\title{
A Spatial and Temporal Gradient of Fgf Differentially Regulates Distinct Stages of Neural Development in the Zebrafish Inner Ear
}

\author{
Shruti Vemaraju, Husniye Kantarci, Mahesh S. Padanad, Bruce B. Riley*
}

Biology Department, Texas A\&M University, College Station, Texas, United States of America

\begin{abstract}
Neuroblasts of the statoacoustic ganglion (SAG) initially form in the floor of the otic vesicle during a relatively brief developmental window. They soon delaminate and undergo a protracted phase of proliferation and migration (transitamplification). Neuroblasts eventually differentiate and extend processes bi-directionally to synapse with hair cells in the inner ear and various targets in the hindbrain. Our studies in zebrafish have shown that Fgf signaling controls multiple phases of this complex developmental process. Moderate levels of Fgf in a gradient emanating from the nascent utricular macula specify SAG neuroblasts in laterally adjacent otic epithelium. At a later stage, differentiating SAG neurons express Fgf5, which serves two functions: First, as SAG neurons accumulate, increasing levels of Fgf exceed an upper threshold that terminates the initial phase of neuroblast specification. Second, elevated Fgf delays differentiation of transit-amplifying cells, balancing the rate of progenitor renewal with neuronal differentiation. Laser-ablation of mature SAG neurons abolishes feedback-inhibition and causes precocious neuronal differentiation. Similar effects are obtained by Fgf5-knockdown or global impairment of Fgf signaling, whereas Fgf misexpression has the opposite effect. Thus Fgf signaling renders SAG development self-regulating, ensuring steady production of an appropriate number of neurons as the larva grows.
\end{abstract}

Citation: Vemaraju S, Kantarci H, Padanad MS, Riley BB (2012) A Spatial and Temporal Gradient of Fgf Differentially Regulates Distinct Stages of Neural Development in the Zebrafish Inner Ear. PLoS Genet 8(11): e1003068. doi:10.1371/journal.pgen.1003068

Editor: Lisa Goodrich, Harvard Medical School, United States of America

Received April 10, 2012; Accepted September 21, 2012; Published November 15, 2012

Copyright: (C) 2012 Vemaraju et al. This is an open-access article distributed under the terms of the Creative Commons Attribution License, which permits unrestricted use, distribution, and reproduction in any medium, provided the original author and source are credited.

Funding: This work was funded by NIH/NIDCD grant R01-DC03806. The funders had no role in study design, data collection and analysis, decision to publish, or preparation of the manuscript.

Competing Interests: The authors have declared that no competing interests exist.

*E-mail: briley@mail.bio.tamu.edu

\section{Introduction}

Neurons of the VIII ${ }^{\text {th }}$ cranial ganglion, or the statoacoustic ganglion (SAG), innervate sensory hair cells in the inner ear. These bipolar neurons relay auditory and vestibular information to the hindbrain. During development, SAG precursors (neuroblasts) originate in the floor of the otic vesicle during a relatively brief window of time. Newly specified neuroblasts soon delaminate from the floor of the otic vesicle before continuing development outside the ear. Neuroblast specification requires the bHLH transcription factor neurogenin1 (neurog1) [1,2]. Expression of neurog1 is transient and is followed by strong upregulation of neurod, which encodes a related bHLH transcription factor required for completing neuronal differentiation [1,3]. After delamination, neuroblasts migrate a short distance to become situated between the hindbrain and otic vesicle and undergo a transient phase of proliferation to expand the precursor population [4-7]. This phase, termed transit-amplification, is characterized by co-expression of neurod and proliferation markers [8]. Neuroblasts eventually exit the cell cycle and differentiate into mature neurons.

Numerous studies suggest a role for Fgf in otic neurogenesis. In chick, Fgflo is expressed in the neurosensory domain of the otic placode and promotes neuroblast specification [4]. Elevating Fgf2 or Fgf8 increases the number of SAG neurons $[9,10]$, though the mechanism of action in these cases has not been determined. In mouse, Fgf3 is also expressed in the neurosensory domain, and SAG development is impaired in Fgf3 null mutants [11]. In zebrafish, $f g f 3$ and $f g f \delta$ are prominently expressed in the developing utricular macula adjacent to the neurogenic domain [12,13], and impairment of $f g f o$ causes a reduction in SAG markers [12,14]. Also in zebrafish, mutations that expand the domain of $f g f 3$ expression in the hindbrain cause a corresponding expansion of anterior markers in the otic vesicle, including markers of the utricular macula and neurogenic domain $[15,16]$. Unfortunately, interpretation of these mutant phenotypes in mouse and zebrafish is clouded because morphogenesis of the inner ear is significantly altered. Additionally, previous studies have not been able to clearly distinguish effects of changing Fgf levels on different stages of SAG development.

Here we study the development of SAG and its regulation by Fgf by conditionally manipulating Fgf signaling levels. We show that Fgf signaling differentially controls distinct stages of otic neurogenesis. A moderate level of Fgf is necessary for the initial specification of neuroblasts in the floor of the otic vesicle, whereas high levels of Fgf inhibit specification. During later stages of SAG development, Fgf5 expressed by mature SAG neurons serves two roles. First, upon accumulation of sufficient mature neurons the phase of specification is terminated. Second, ongoing Fgf signaling delays the differentiation of SAG precursor cells. This ensures maintenance of progenitors and steady production of an appropriate number of mature neurons. 


\section{Author Summary}

Neurons of the statoacoustic ganglion (SAG), which innervate the inner ear, are derived from neuroblasts originating from the floor of the otic vesicle. Neuroblasts quickly delaminate from the otic vesicle to form dividing progenitors, which eventually differentiate into mature neurons of the SAG. Fgf has been implicated in initial neuroblast specification in multiple vertebrate species. However, the role of Fgf at later stages remains uncertain, because previous studies have not been able to evaluate the effects of changing levels of Fgf, nor have they been able to clearly distinguish the effects of Fgf at different stages of SAG development. We have combined conditional loss of function, misexpression, and laser-ablation studies in zebrafish to elucidate how graded Fgf coordinates distinct steps in SAG development. Initially moderate Fgf in a spatial gradient specifies neuroblasts within the otic vesicle. Later, mature SAG neurons express Fgf5 and, as additional neurons accumulate outside the otic vesicle, rising levels of Fgf terminate further specification. Elevated Fgf also slows maturation of progenitors, maintaining a stable progenitor pool in which growth and differentiation are evenly balanced. This feedback facilitates steady production of new neurons as the animal grows through larval and adults stages.

\section{Results}

\section{Development of the statoacoustic ganglion (SAG)}

The paradigm for otic neurogenesis, as formalized in several recent reviews $[17,18]$, involves a sequential process of specification, delamination, proliferative expansion and differentiation of precursor cells to form the mature SAG. The general features of this process appear to be conserved in zebrafish, shown schematically in Figure 1A. In zebrafish, SAG neuroblasts are initially specified in the floor of the late placode/nascent vesicle as early as $16 \mathrm{hpf}(14$ somites) and express neurog1 [1,19]. Neuroblasts begin to delaminate and accumulate outside the otic vesicle by $17 \mathrm{hpf}$. The reiterative process of specification and delamination peaks at $24 \mathrm{hpf}$, continues at a more moderate pace through $30 \mathrm{hpf}$ (Figure 1D), then declines sharply and stops entirely by 42 hpf [20]. Expression of neurog 1 is only transient. As neuroblasts delaminate they lose expression of neurog1 and initiate expression of neurod $[1,21]$. At this point SAG precursors enter a phase of transit-amplification, as shown by co-labeling with neurod expression and BrdU incorporation (Figure 1E, 1F). neurod ${ }^{+}$cells continue to proliferate through at least 4 days post fertilization (dpf), the latest stage examined (Figure 1F). Surprisingly, staining with anti-phospho histone $\mathrm{H} 3$ shows that there are typically only 1-2 mitotic cells in the SAG at any time between 24 and $50 \mathrm{hpf}$ (Figure 1G, and data not shown), indicating that transitamplifying cells cycle relatively slowly. Summing neurod ${ }^{+}$cells in serial sections revealed that the number of transit-amplifying cells remains relatively constant after $30 \mathrm{hpf}$, with a transient peak at $48 \mathrm{hpf}$ followed by a return to steady state of 180-200 cells through $78 \mathrm{hpf}$ (Figure 1B). As precursor cells begin to differentiate they exit the cell cycle and lose expression of neurod (Figure 1E, 1F) and initiate expression of isl1/2 genes (Figure 1H, 1I) [3]. The first mature $\mathrm{Isll}^{+}$neurons appear by $20 \mathrm{hpf}$ and almost immediately begin to extend processes to peripheral and central targets (data not shown). Co-staining for BrdU and Gfp in isl2b:Gfp transgenic embryos [22] confirms that relatively few mature neurons incorporate BrdU (Figure 1H). The number of $\mathrm{Isll}^{+}$neurons increases linearly at a rate of 2-2.5 neurons per hour through at least $72 \mathrm{hpf}$, despite the cessation of specification and delamination at $42 \mathrm{hpf}$ (Figure 1C). The steady increase in mature neurons after $42 \mathrm{hpf}$ presumably reflects ongoing differentiation from the slowly cycling pool of transit-amplifying cells. The slow mitotic rate amongst precursors presumably counterbalances production of new neurons, thereby maintaining a relatively stable transit-amplifying pool.

To clarify the spatial relationship between transit-amplifying and mature SAG cells, we examined sections co-stained for neurod and Isl1 (Figure 1I-1M). The most mature neurons accumulate in immediate contact with the ventromedial surface of the ear, while neurod $^{+}$cells undergoing transit-amplification reside more distally (Figure 1L, 1M). By $36 \mathrm{hpf}$ the SAG also develops a more complex spatial distribution, forming three distinct regions along the anterior-posterior axis: The anterior-most region abuts the front end of the otic vesicle and contains only neurod ${ }^{+}$precursors (Figure 1K), although mature neurons accumulate in this region at later stages (see below). The middle region forms a broad mass spreading mediolaterally beneath the utricular region and contains complementary domains of neurod ${ }^{+}$cells and $\mathrm{Isll}^{+}$cells (Figure 1L). The posterior-most region forms as a narrow finger of Islet $^{+}$cells and abutting neurod ${ }^{+}$cells extending along the medial surface of the otic vesicle to the level of the saccular macula (Figure 1M). Segregation of neurons into these three AP domains reflects emergence of the topological pattern of innervation of the inner ear: Specifically, Sapède and Pujades [23] reported that anteroventral SAG neurons (corresponding to the anterior and middle regions reported here) predominantly innervate the utricular macula and to a lesser degree anterior and lateral cristae, whereas posterior-medial SAG neurons (corresponding to the posterior region reported here) predominantly innervate the saccular macula and to a lesser degree the posterior crista.

\section{A general model of SAG regulation and manipulation of Fgf signaling}

The data presented in subsequent sections support a model in which changing levels of Fgf differentially affect SAG development: Initially, moderate Fgf from nearby cells promotes neuroblast specification in the otic vesicle. Subsequently, Fgf levels rise in part because mature SAG neurons specifically express Fgf5 and accumulate just outside the otic vesicle (Figure 2). Elevated Fgf then terminates further specification/delamination and also inhibits maturation of transit-amplifying precursors. Manipulation of Fgf to test this model was achieved by specifically knocking down $f g f 5$ (described below) and more generally by using two heat shock inducible transgenic lines, $h s: f f g$ and $h s: d n f g f r 1$ (dominant-negative Fgf receptor), to increase or decrease Fgf signaling, respectively $[24,25]$. To document the efficacy of these transgenic lines, we examined expression of the Fgf-feedback gene etv $5 b$ (previously erm) [26,27] following activation of the transgenes. Strong activation of $h s: f g f^{\circ}$ by heat shocking embryos at $24 \mathrm{hpf}$ $\left(30\right.$ minutes at $39^{\circ} \mathrm{C}$ ) led to a detectable increase in etv $5 b$ levels by the end of the heat shock period (not shown), with maximal etv $5 b$ seen throughout the embryo by $26 \mathrm{hpf}$ (Figure 3B, 3E). etv $5 b$ levels remained elevated through $30 \mathrm{hpf}$ (Figure $3 \mathrm{H}$ ) and subsequently returned to normal. In contrast, strong activation of $h s: \operatorname{dnfg} f r l$ at $24 \mathrm{hpf}\left(30\right.$ minutes at $\left.38^{\circ} \mathrm{C}\right)$ led to marked reduction of etv $5 b$ expression throughout the embryo by $25 \mathrm{hpf}$ (not shown), and complete loss by 26 hpf (Figure 3C, 3F). Expression first began to return by $36 \mathrm{hpf}$, though levels were still well below normal at that time (Figure 3I). These transgenes were subsequently used to assess the effects of changing Fgf signaling levels at different stages of SAG development. 


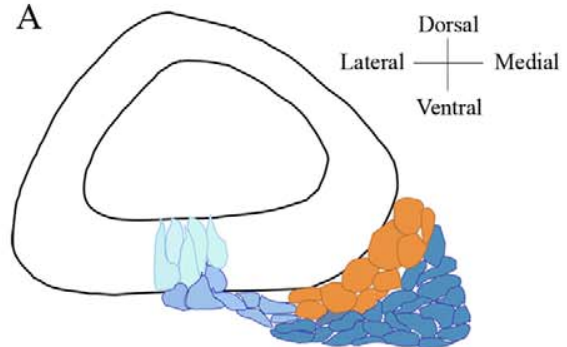

1. Specification 3. Transit-amplification 2. Delamination 4. Mature neurons
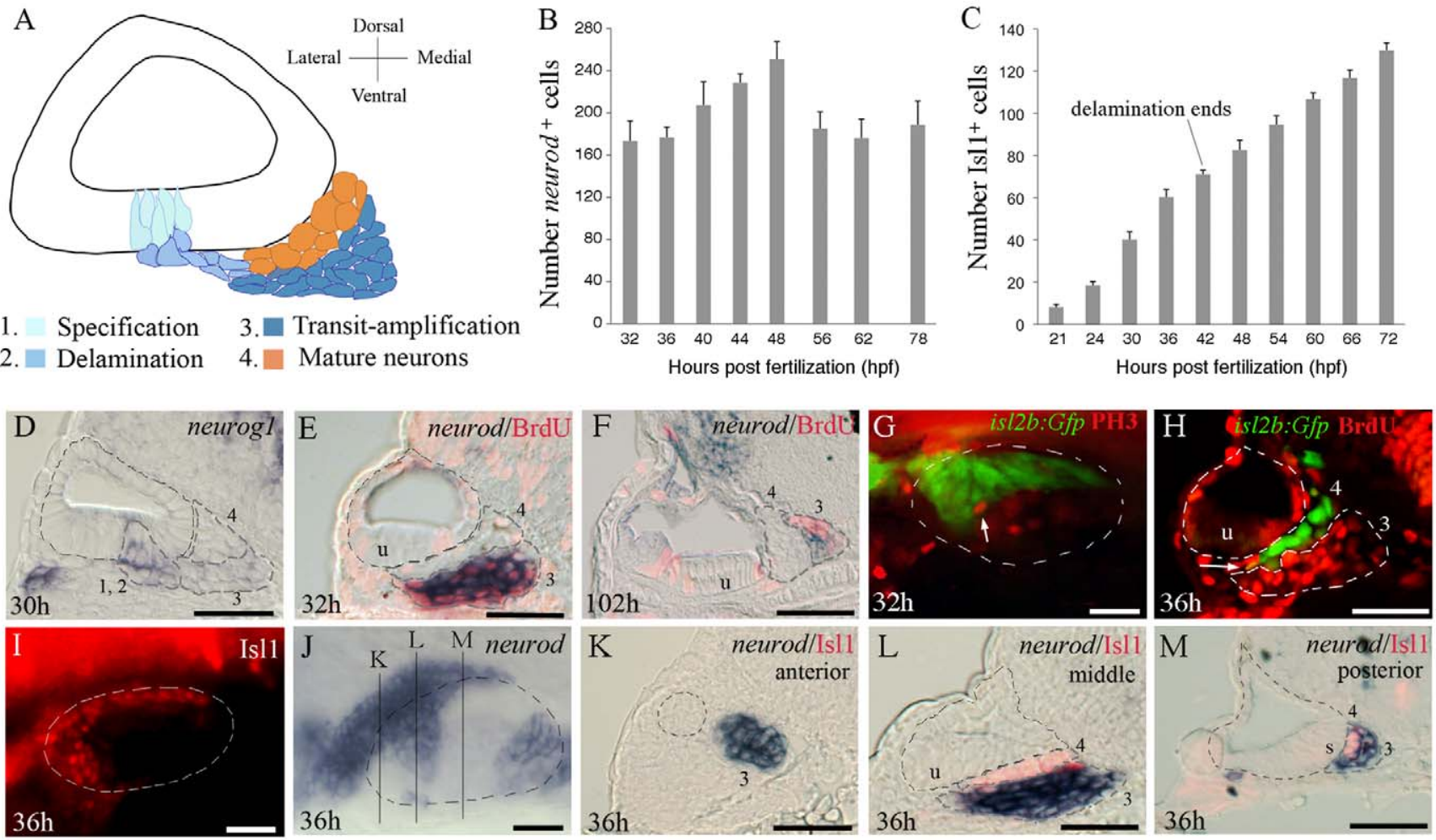

Figure 1. Development of Statoacoustic Ganglion (SAG). (A) Illustration showing the various stages of SAG development. Neuronal precursors (neuroblasts) are specified (1) and delaminate from (2) the floor of the otic vesicle. Neuroblasts undergo a phase of transit-amplification (3) wherein they migrate to a position between the otic vesicle and hindbrain as they continue to proliferate. Neuroblasts finally differentiate into mature neurons (4). (B) Total number of delaminated neurod ${ }^{+}$cells within the SAG counted from serial sections at the indicated times (mean \pm standard deviation, $n=2$ or greater for each time point). (C) total number of Islet-1-positive SAG neurons at the indicated times (mean of total number \pm standard deviation, $\mathrm{n}=20$ for each time point). (D) neurog1 expression at $30 \mathrm{hpf}$. (E, F) Co-staining for neurod (blue) and BrdU (red) in embryos exposed to BrdU for 6 hours starting at $26 \mathrm{hpf}(\mathrm{E})$ and $96 \mathrm{hpf}(\mathrm{F})$, and then fixed at $32 \mathrm{hpf}$ and $102 \mathrm{hpf}$, respectively. (G) Co-staining for is/2b:Gfp (green) and phospho-histone H3 (PH3, red) at 32 hpf. Only one mitotic cell (arrow) is seen in the vicinity of the SAG. (H) Co-staining for Islet1 (green) and $\mathrm{BrdU}$ (red) at $36 \mathrm{hpf}$. Only one double-stained cell is visible (arrow). (I-M) Expression of neurod (blue) and Islet-1 (red) at $36 \mathrm{hpf}$. Mature neurons are labeled with Islet-1 (I) and delaminated progenitor cells express neurod (J). Positions of section-planes in K-M are indicated in (J). (K-L) Transverse sections passing through the anterior (K), middle (L) and posterior $(\mathrm{M})$ regions of the SAG show mostly complementary patterns of neurod and Islet-1. The outer edge of the otic vesicle is outlined in all panels. SAG cells in stages 1-4 of development are indicated in sections, and the position of the utricular macula $(\mathrm{u})$ is indicated. Images of whole-mount specimens $(\mathrm{G}, \mathrm{I}, \mathrm{J})$ show dorsolateral $(\mathrm{G}, \mathrm{I})$ and dorsal $(\mathrm{J})$ views with anterior to the left. Images of transverse sections $(\mathrm{C}-\mathrm{F}, \mathrm{H}, \mathrm{K}-\mathrm{M})$ show dorsal to the top and lateral to the left. Scale bar, $25 \mu \mathrm{m}$.

doi:10.1371/journal.pgen.1003068.g001

\section{Fgf regulates SAG specification in a dose-dependent manner}

Several Fgfs expressed in tissues near the developing SAG have been implicated in establishing a neurogenic domain in the ear [28]. In zebrafish, $f g f 3$ is expressed in the adjacent hindbrain through placodal stages and later helps initiate expression of $f g f 3$ and $f g f o$ in the nascent utricular macula by $18 \mathrm{hpf}$ [15]. We have hypothesized that sensory-neural patterning is spatially coordinated by a lateral gradient of Fgf, with high levels initiating sensory development in the medial half of the otic placode - e.g. closest to the Fgf source [13], and lower levels specifying the neurogenic domain in laterally adjacent otic epithelium. We previously documented a stringent requirement for Fgf in sensory development [13] and here we focused on the requirement for Fgf in neurogenic specification. To bypass the early requirements of Fgf during otic induction we used the chemical inhibitor, SU5402, to block Fgf signaling at later stages of otic development. Embryos treated with $100 \mu \mathrm{M}$ SU5402 from $14 \mathrm{hpf}-18$ hpf showed a strong reduction in neurog1 expression (Figure 4A, 4B). Likewise, impairment of Fgf signaling by strongly activating hs:dnfgfrl [25] $\left(38^{\circ} \mathrm{C}\right.$ for 30 minutes) showed similar results (Figure $4 \mathrm{C}$ ). Blocking Fgf from this early stage caused widespread cell death at later stages, precluding analysis of SAG maturation. Nevertheless, these data confirm that normal specification of the neurogenic domain requires Fgf signaling. To test the hypothesis that SAG neuroblasts are specified by a specific lower level of $\mathrm{Fgf}$ in a signaling gradient, we manipulated Fgf levels using hs: $f g f o$ [25]. The level of $h s: f g f o$ activity can be adjusted by heat shocking at different temperatures [29]. To provide a broad shelf of low Fgf signaling, embryos were incubated at $35^{\circ} \mathrm{C}$ from $18 \mathrm{hpf}$ to $24 \mathrm{hpf}$. This caused a marked upregulation and expansion of neurog1 expression (Figure 4D, 4E). Additionally, there was a notable increase in the number of delaminating neuroblasts as seen by $h m \times 3$ expressing cells leaving the vesicle (Figure 4F, 4G). By $42 \mathrm{hpf}$, the number of $\mathrm{Isll}^{+}$cells in the mature SAG had increased by $37 \%$ over the control (Figure 4H, 63 $46.0 \mathrm{Isll}^{+}$cells in control embryos compared to $86 \pm 3.6$ in $h s: f g f o$ transgenic embryos, $\mathrm{n}=15$ ). To evaluate the effects of a higher level of Fgf, $h s: f g f o$ embryos were maximally induced by heat shocking them at $39^{\circ} \mathrm{C}$ for 30 minutes beginning at $18 \mathrm{hpf}$. Under these conditions, neurog1 expression was reduced for several hours following heat shock but recovered to near 

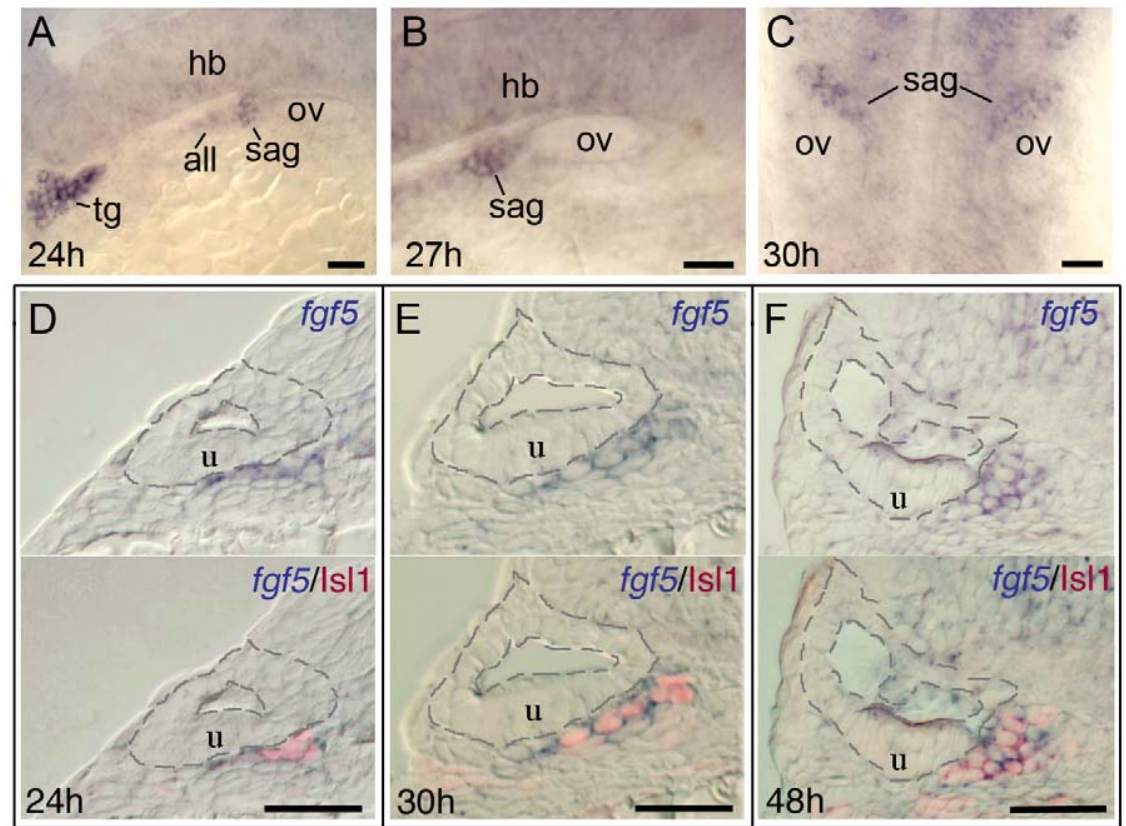

Figure 2. Mature SAG neurons express fgf5. (A-C) Wholemount embryos showing lateral views of fgf5 expression at $24 \mathrm{hpf}(\mathrm{A})$ and $27 \mathrm{hpf}(\mathrm{B})$ and a dorsal view at $30 \mathrm{hpf}$ (C). During these stages, fgf5 expression marks the trigeminal ganglion (tg), anterior lateral line ganglion (all) and SAG, and there is also weak diffuse expression in the developing hindbrain (hb). There is no detectable staining in the otic vesicle (ov). (D-F) Transverse sections (dorsal to the top and lateral to the left) of specimens co-stained for fgf5 (blue) and Islet-1 (red) at $24 \mathrm{hpf}(\mathrm{D}), 30 \mathrm{hpf}(\mathrm{E})$ and $48 \mathrm{hpf}(\mathrm{F})$. Sections pass through the middle portion of the SAG at the level of the utricular macula (u). The inner and outer surfaces of the otic vesicle are outlined. Co-labeling confirms that $f g f 5$ expression in the SAG is restricted to mature neurons. Scale bar, $25 \mu \mathrm{m}$. During mid-somitogenesis stages fgf5 is diffusely expressed throughout the neural tube and strongly marks the developing trigeminal ganglion (not shown). doi:10.1371/journal.pgen.1003068.g002

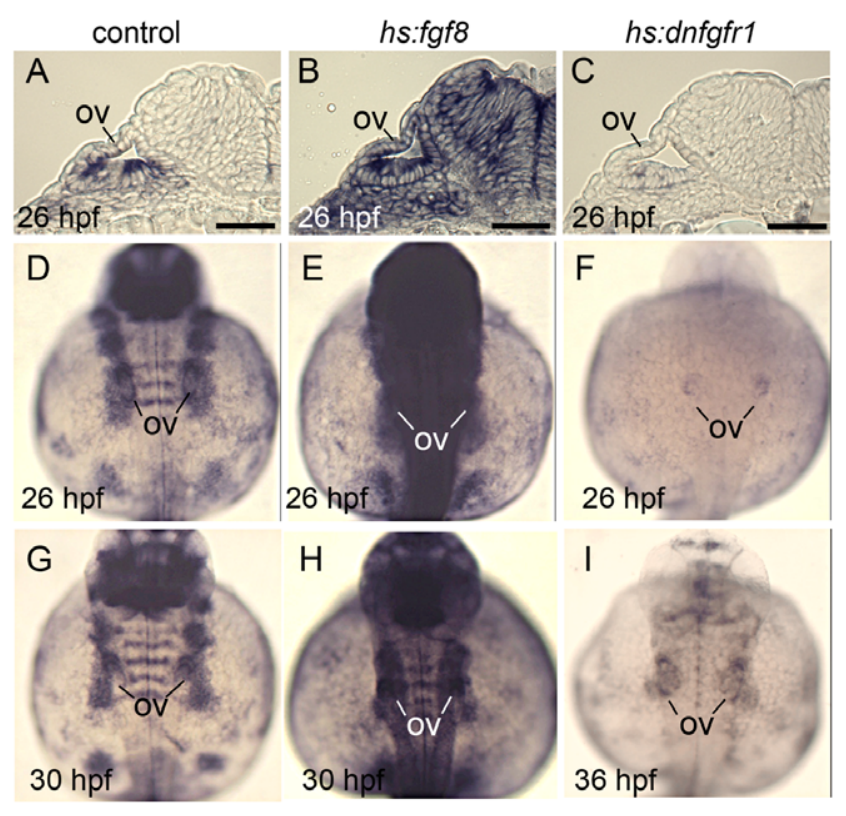

Figure 3. Effects of transgene activation on expression of the Fgf reporter etv $5 b$. All embryos were heat shocked for 30 minutes beginning at $24 \mathrm{hpf}$. Wild-type and hs:fgf8 embryos were heat shocked at $39^{\circ} \mathrm{C}$ and $h s:$ dnfgfr 1 embryos were heat shocked at $38^{\circ} \mathrm{C}$. (A-C) Cross sections showing etv5b expression in wild-type (A), hs:fgf (B) and hs:dnfgfr 1 (C) embryos at 26 hpf. (D-I) Dorsal views (anterior up) of wholemounts showing etv5b expression in wild-type $(D, G)$, hs:fgf8 $(E, H)$ and $h$ s:dnfgfr $1(F, I)$ embryos at the indicated times. The otic vesicle (ov) is marked. Expression of etv5b remains elevated in hs:fgf8 embryos for at least 6 hours after heat shock, whereas etv $5 b$ expression is downregulated in hs:dnfgfr1 for at least 12 hours after heat shock. Scale bar, $25 \mu \mathrm{m}$.

doi:10.1371/journal.pgen.1003068.g003

normal by $24 \mathrm{hpf}$ (data not shown). However, the number of mature $\mathrm{Isll}^{+}$neurons at $42 \mathrm{hpf}$ was reduced by $20 \%\left(51 \pm 4.2 \mathrm{Isll}^{+}\right.$ cells, $n=15$; Figure $4 \mathrm{H}$ ). As summarized in Table 1 , these data support the idea that Fgf acts in a concentration-specific manner, with lower levels promoting neuroblast specification and higher levels inhibiting specification.

Because the rate of neuroblast specification and delamination peaks at $24 \mathrm{hpf}$, we examined the effects of Fgf misexpression during this stage. As before, maximal activation of $h s: f g f\left(39^{\circ} \mathrm{C}\right)$ at $24 \mathrm{hpf}$ reduced expression of neurog 1 in the ear by $30 \mathrm{hpf}$ (Figure 4I, 4J). However, in contrast to earlier stages, low level activation of $h s: f g f 8\left(35^{\circ} \mathrm{C}\right)$ at $24 \mathrm{hpf}$ reduced neurog 1 expression by $30 \mathrm{hpf}$ (data not shown). Fully blocking Fgf by strong activation of hs:dnfgfr $1\left(38^{\circ} \mathrm{C}\right)$ at $24 \mathrm{hpf}$ also diminished neurog 1 expression by $30 \mathrm{hpf}$ (data not shown), in keeping with the requirement for Fgf in neuroblast specification. However, weak attenuation of Fgf signaling by activating $h s: d n f g f r l$ at a low level $\left(35^{\circ} \mathrm{C}\right.$ for 2 hours followed by incubation at $33^{\circ} \mathrm{C}$ ) expanded the neurog 1 expression domain at $30 \mathrm{hpf}$ (Figure 4K). Overall these data (summarized in Table 1) support the hypothesis that a specific low-to-moderate level of Fgf promotes neuroblast specification at both early and later stages, and either a high level of Fgf signaling or complete blockage of Fgf signaling impairs this process. At later stages, however, the process of specification becomes increasingly 


\section{Neuroblast specification}

\section{I. $\downarrow$ Fgf $14-18 \mathrm{hpf}$, examine neurog1 at $18 \mathrm{hpf}$.}

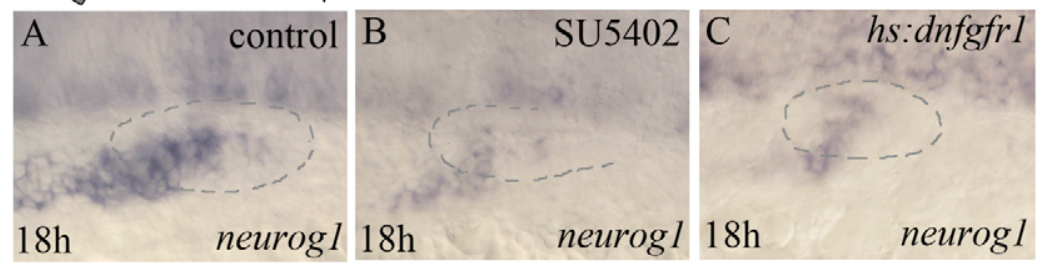

II. $\uparrow$ Fgf 18-24 hpf, examine neurog1 \& $h m \times 3$ at $24 \mathrm{hpf}$.
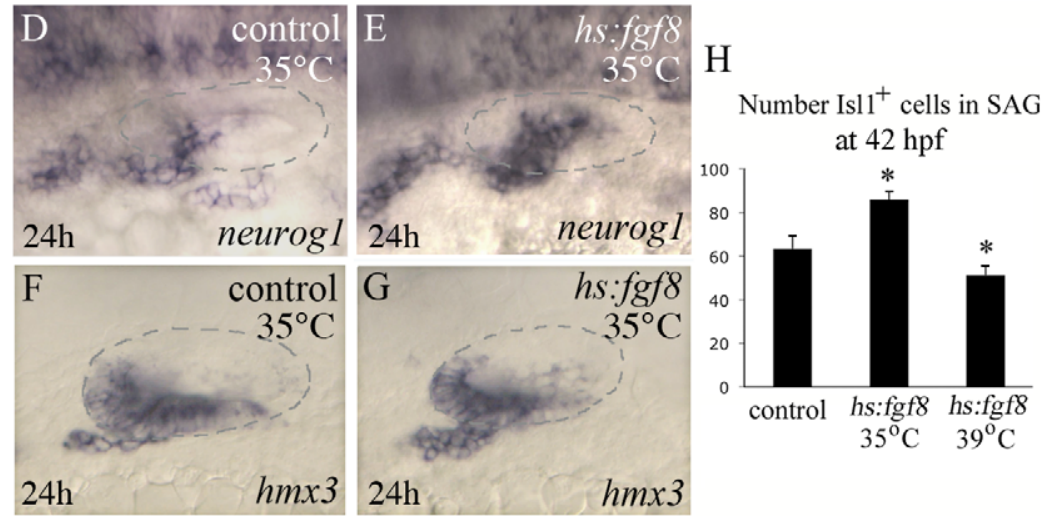

$24 \mathrm{~h}$

$h m x 3$

III. $\downarrow$ or $\boldsymbol{\uparrow}$ Fgf at $24 \mathrm{hpf}$, examine neurog1 at $30 \mathrm{hpf}$.

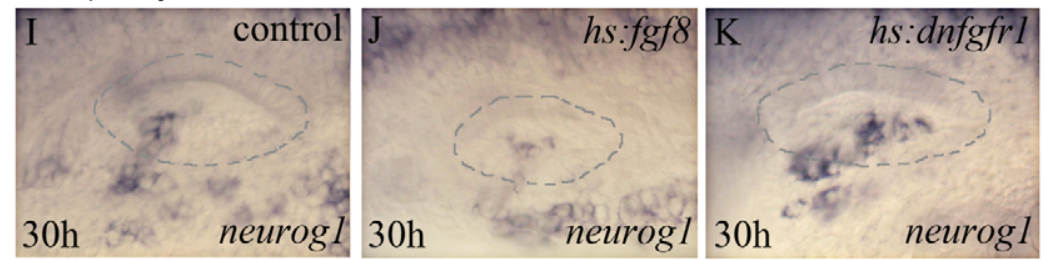

Figure 4. Fgf regulates neuroblast specification. The icon at the top of the figure indicates that analysis focuses on initial stages of neuroblast formation, as normally marked by neurog1 expression. Experimental manipulations in groups I, II and III are briefly summarized at the tops of the corresponding data panels. (A-C) Experiment I, neurog 1 expression at $18 \mathrm{hpf}$ in a control (A), SU5402 inhibitor treated (B) and hs:dnfgfr 1/+ transgenic embryo heat shocked for 30 minutes at $38^{\circ} \mathrm{C}$ beginning at $14 \mathrm{hpf}$. Blocking Fgf strongly reduces expression of neurog1. (D-G) Experiment II, expression of neurog1 (D-E) and $h m \times 3$ (F-G) in control and hs:fgf8/+ embryos heat shocked at $35^{\circ} \mathrm{C}$ for 6 hours, from $18 \mathrm{hpf}$ until $24 \mathrm{hpf}$. This regimen results in weak overexpression of Fgf8, which at this stage enhances expression of neurog1. $(\mathrm{H})$ Experiment II, total number of Islet1-positive cells in the SAG (mean and standard deviation, $n=15$ ) at $42 \mathrm{hpf}$ following heat shock activation of $h$ s:fgf8 at 18 hpf at indicated temperatures. Weak misexpression of Fgf8 $\left(35^{\circ} \mathrm{C}\right)$ increases production of SAG neurons whereas strong misexpression of Fgf8 $\left(39^{\circ} \mathrm{C}\right)$ reduces production of SAG neurons. ${ }^{*} p<0.001$ in comparison to the control, analyzed with Student's $t$ test. (I-K) Group III, neurog1 expression at 30 hpf following heat shock at 24 hpf in control embryos (I), hs:fgf8/+ embryos heat shocked at $39^{\circ} \mathrm{C}$ for 30 minutes to strongly over-express Fgf $(\mathrm{J})$ and $h s: d n f g f r 1 /+$ embryos heat shocked for 2 hours at $35^{\circ} \mathrm{C}$ and then shifted to $33^{\circ} \mathrm{C}$ to maintain low level inhibition of Fgf signaling (K). At this stage, weak impairment of Fgf enhances neurog 1 expression, consistent with the idea that Fgf levels normally increase during development and become inhibitory for neuroblast specification. All images show dorsolateral views with anterior to the left, and the otic vesicle is outlined. doi:10.1371/journal.pgen.1003068.g004

sensitive to inhibition by elevated Fgf. This likely reflects the finding that the level of Fgf increases during development, as described in the next section.

\section{fgf5 from mature neurons inhibits neuroblast specification}

Because SAG specification becomes increasingly sensitive to inhibition by elevated Fgf, we hypothesized that the process of neuroblast specification is normally terminated by a developmental increase in local Fgf signaling. To explore this possibility, we surveyed expression of all known $f g f$ genes in zebrafish and identified $f g f 5$ as a strong candidate for a feedback regulator of SAG development. During mid-somitogenesis stages $f g f 5$ is diffusely expressed throughout the neural tube and strongly marks the developing trigeminal ganglion (not shown). As mentioned above, $f g f 5$ shows relatively specific expression in mature SAG neurons, and several other cranial ganglia, by $24 \mathrm{hpf}$ and this pattern is maintained through at least $48 \mathrm{hpf}$ (Figure 2). No expression is detected in the otic vesicle or other nearby tissues. We tested the role of Fgf5 by injecting morpholino oligomers to block translation $(f g f 5 t b-\mathrm{MO})$ or to disrupt splicing at the intron 1exon2 splice junction (fof 5 ile2-MO). Injection of either MO yielded identical phenotypes: Morphants showed highly specific 
Table 1. Effects of altering Fgf on neuroblast specification.

\begin{tabular}{lll}
\hline & & \\
\hline Condition and stage & neurog1 domain & Figure \\
\hline hs:fgf8-high $\left(39^{\circ} \mathrm{C}\right), 18 \mathrm{hpf}$ & reduced $20 \mathrm{hpf}$ & - \\
hs:fgf8-low $\left(35^{\circ} \mathrm{C}\right), 18 \mathrm{hpf}$ & enlarged $24 \mathrm{hpf}$ & $4 \mathrm{E}$ \\
hs:dnfgfr1-high $\left(38^{\circ} \mathrm{C}\right), 14 \mathrm{hpf}$ & reduced $18 \mathrm{hpf}$ & $4 \mathrm{C}$ \\
SU5402 $(100 \mu \mathrm{M}), 14 \mathrm{hpf}$ & reduced $18 \mathrm{hpf}$ & $4 \mathrm{~B}$ \\
hs:fgf8-high $\left(39^{\circ} \mathrm{C}\right), 24 \mathrm{hpf}$ & reduced $30 \mathrm{hpf}$ & $4 \mathrm{~J}$ \\
hs:fgf8-low $\left(35^{\circ} \mathrm{C}\right), 24 \mathrm{hpf}$ & reduced $30 \mathrm{hpf}$ & - \\
hs:dnfgfr1-high, $24 \mathrm{hpf}$ & reduced $30 \mathrm{hpf}$ & $4 \mathrm{~K}$ \\
hs:dnfgfr1-low $\left(35^{\circ} \mathrm{C}\right), 24 \mathrm{hpf}$ & enlarged $30 \mathrm{hpf}$ & - \\
fgf5-MO, 1-cell & enlarged $30,36 \mathrm{hpf}$ & $6 \mathrm{E}-6 \mathrm{G}$ \\
fgf5-MO+hs:fgf8 $\left(39^{\circ} \mathrm{C}\right), 24 \mathrm{hpf}$ & normal $36 \mathrm{hpf}$ & $6 \mathrm{H}$ \\
Ablate mature SAG $22,25 \mathrm{hpf}$ & enlarged $30 \mathrm{hpf}$ & $6 \mathrm{~L}$ \\
\hline doi:10.1371/journal.pgen.1003068.t001 & &
\end{tabular}

and fully penetrant enhancement of SAG specification and maturation, as described below, but otherwise there were no other detectable changes in embryo morphology nor was there a detectable increase in cell death. For most experiments reported here, we show results obtained with fgf5ile $2-\mathrm{MO}$, which proved to be highly effective in reducing mature $f g f 5$ transcript levels (Figure 5A, 5B).

To address the role of $f g f 5$ in neuroblast specification we examined neurog 1 expression at various stages in $f g f 5$ morphants. At $24 \mathrm{hpf}$ no obvious difference was observed between $f g f 5$ morphants and control embryos (not shown). By $30 \mathrm{hpf}$, however, neurog1 expression was dramatically expanded in $f g f 5$ morphants (Figure 6A, 6B, 6E, 6F), including a pronounced mediolateral expansion of neurog1 in the floor of the otic vesicle (Figure $6 \mathbf{B}, 6 \mathbf{F}$ ). Normally, neuroblast specification declines dramatically after 30 hpf $[1,20]$ (Figure 6C). However, fgf5 morphants continued to show abundant neurog1-positive cells at $36 \mathrm{hpf}$, indicating a prolonged phase of robust specification and delamination (Figure 6G). Neuroblast specification/delamination finally ceased by $48 \mathrm{hpf}$ in $f g f 5$ morphants (not shown). Knockdown of $f g f 5$ appeared to affect SAG development in a highly specific manner, as other regional markers in the otic vesicle were expressed normally and development of sensory hair cells was also normal at $32 \mathrm{hpf}$ (Figure 7). Additionally, the $f g f 5$ morphant phenotype was rescued by strong activation of $h s: f g f 8\left(39^{\circ} \mathrm{C}\right)$ at $24 \mathrm{hpf}$ such that neuroblast specification returned to normal (Figure 6D, 6H). Such rescue supports the idea that neuroblast specification relies on a proper balance of Fgf signaling, with the morpholino and transgene counter-balancing each other. Overall, these data (summarized in Table 1) support the hypothesis that mature

A

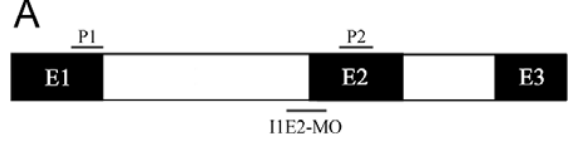

B control fgf5-MO

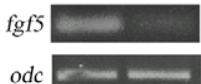

Figure 5. Efficacy of $f g f 5$ splice-blocking MO. (A) Schematic of $f g f 5$ mRNA showing intron-exon structure (not to scale). Binding sites for splice-blocking morpholino at intron1-exon2 junction (I1E2-MO) and PCR primers for RT-PCR (forward P1, reverse P2) are shown. (B) RT-PCR results showing the efficacy of 11E2-MO. fgf5 transcript levels are severely reduced in fgf5 morphants at $24 \mathrm{hpf}$. odc transcript level was used as a constitutive control.

doi:10.1371/journal.pgen.1003068.g005
SAG cells become a source of elevated Fgf, which eventually exceeds a signaling threshold that serves to terminate neuroblast specification in a timely manner.

To test this model in another way, mature neurons marked by isl2b:gfp transgene expression [22] were killed by serial laserablation at $22 \mathrm{hpf}$ and $25 \mathrm{hpf}$ (Figure 6I, 6J) and neurog1 expression was examined at $30 \mathrm{hpf}$. Expression of neurogl was expanded on the ablated side relative to the unablated (contralateral) side (Figure 6K, 6L, Table 1). Together, these data support the notion that as mature neurons expressing $f g f 5$ accumulate within the SAG, overall levels of Fgf signaling increase and as a result neuroblast specification is terminated. This also explains the increased susceptibility to misexpression of Fgf8 after $24 \mathrm{hpf}$, as described above.

\section{Fgf regulates the balance between transit-amplification} and differentiation

We next examined the effects of Fgf on post-delamination stages of SAG development. In these experiments heat shock transgenes were activated at high levels $\left(38-39^{\circ} \mathrm{C}\right)$ at $24 \mathrm{hpf}$ and the effects on neurod $^{+}$(transit-amplifying) and $\mathrm{Isll}^{+}$(mature) populations were examined at $36 \mathrm{hpf}$ and $48 \mathrm{hpf}$. Summing neurod ${ }^{+}$cells in serial sections of control embryos indicated that there are approximately 200-250 transit-amplifying cells in the SAG at these stages (Figure 1B). Because this approach proved laborious and was prone to occasional loss of tissue sections, changes in the neurod domain were assessed by measuring mean cross-sectional areas in the three AP regions of the SAG in transgenic and control embryos.

Strong activation of $h s: f g f o$ at $24 \mathrm{hpf}\left(39^{\circ} \mathrm{C}\right.$ for 30 minutes $)$ increased the neurod ${ }^{+}$precursor domain by $31 \%$ in the largest, middle region of the SAG at $36 \mathrm{hpf}$ (Figure 8A, 8B, 8I). A similar trend was observed in the anterior region, although the difference was not statistically significant (Figure 8I). Under these conditions, the smallest, posterior part of the SAG was truncated in $h s: f f f$ embryos and therefore was nearly devoid of neurod ${ }^{+}$cells in most specimens (Figure 8I). This is possibly because the posterior SAG forms later and elevated Fgf prematurely terminates specification of neuroblasts that might otherwise contribute to this region. Despite, the increased population of transit-amplifying cells in the middle region, the total number of $\mathrm{Isll}^{+}$neurons in the SAG was reduced in $h s: f g f o$ embryos by $30 \%$ at $36 \mathrm{hpf}$ (Figure $8 \mathrm{E}, 8 \mathrm{~F}, 8 \mathrm{~J}$ ) and the hourly rate of neuron production between $24 \mathrm{hpf}$ and $36 \mathrm{hpf}$ was reduced by half (Figure $8 \mathrm{~K}$ ). For loss of function studies, $h s: d n f g f r l$ was activated at $24 \mathrm{hpf}\left(38^{\circ} \mathrm{C}\right.$ for 30 minutes $)$ to impose a strong block to Fgf signaling. This resulted in a decrease of $26 \%$ in the neurod ${ }^{+}$domain in the middle region at $36 \mathrm{hpf}$, and a decrease of $50 \%$ in the posterior region (Figure 8C, 8I). Again, the anterior region showed a similar but non-significant trend. Under the same conditions, there was a $30 \%$ increase in the total number of mature $\mathrm{Is} 1^{+}$SAG neurons (Figure 8G, 8J). The relative effects of $h s: f g f 8$ and $h s: \operatorname{dnfg} f r 1$ on the transit-amplifying population persisted through at least $48 \mathrm{hpf}$ (Figure 8L). Differences in the total number of mature neurons also persisted at $48 \mathrm{hpf}$ (Figure 8M). However, most of the differences seen at $48 \mathrm{hpf}$ appeared to reflect changes occurring before $36 \mathrm{hpf}$ because the rate of production of new $\mathrm{Isll}^{+}$neurons after 36 hpf was nearly normal in $h s: f g f o$ and $h s: d n f g f r 1$ embryos (compare Figure $8 \mathrm{~K}, 8 \mathrm{~N}$ ). This presumably reflects the transient nature of transgene activity and gradual reestablishment of normal SAG regulation. Note that under the conditions used here, we detected little or no cell death in the transit-amplifying or mature regions of the SAG as shown by staining with Acridine Orange or anti-Caspase 3 antibody (not shown). Likewise, we detected no changes in the number of mitotic cells in the SAG, nor in the 


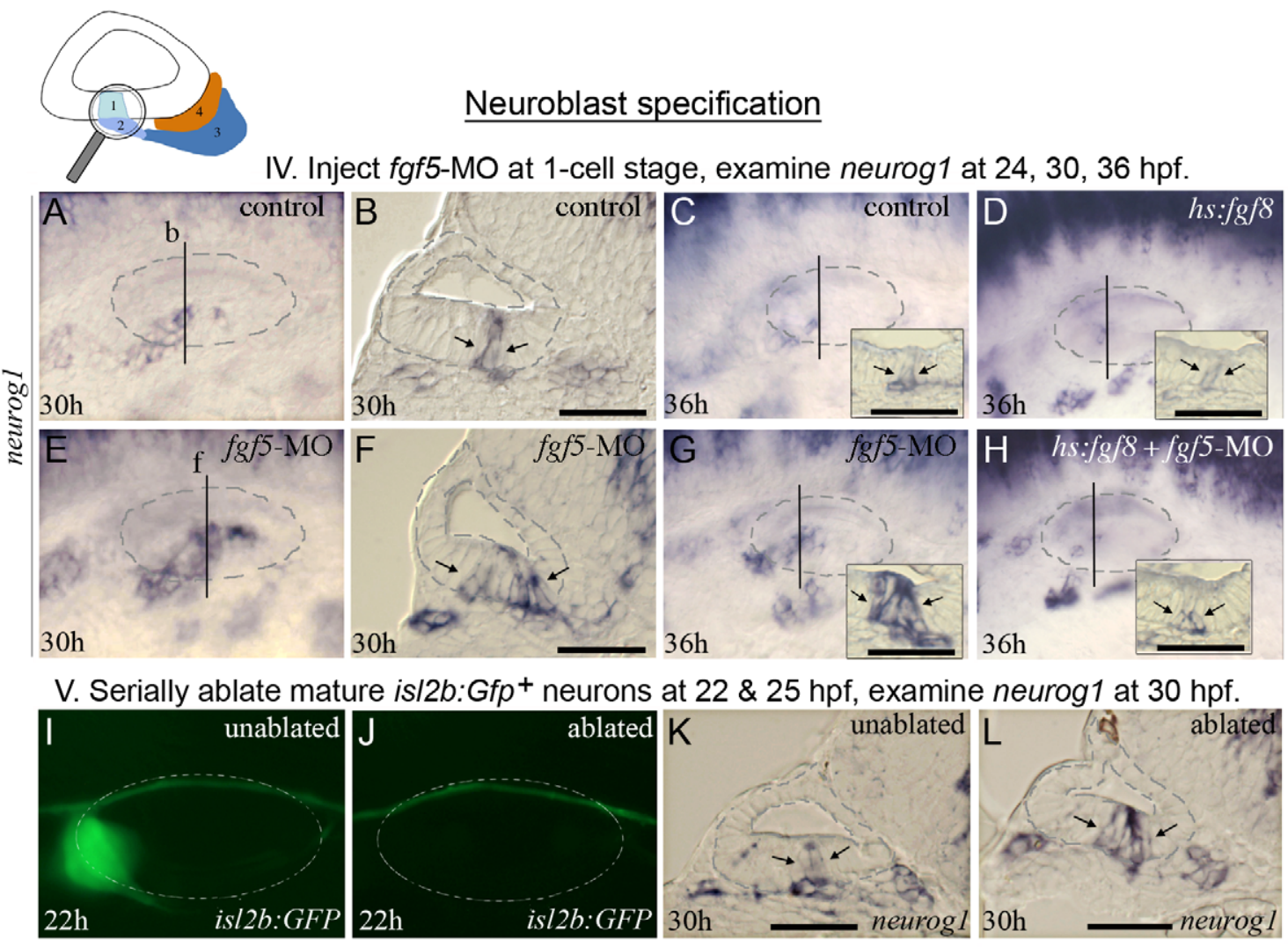

Figure 6. fgf5 from mature neurons terminates the phase of neuroblast specification. The icon at the top of the figure indicates that analysis focuses on initial formation of neuroblasts. Experimental manipulations in groups IV and $\mathrm{V}$ are briefly summarized at the tops of the corresponding data panels. (A-H) Expression of neurog1 in control embryos (A-C), a hs:fgf8 embryo (D), fgf5 morphants (E-G), and a hs:fgf8 embryo injected with fgf5-MO $(\mathrm{H})$ at the indicated stages. Transgenic embryos $(\mathrm{D}, \mathrm{H})$ were heat shocked for 30 minutes at $39^{\circ} \mathrm{C}$ beginning at 24 hpf. Vertical lines in $(A, C-E, G, H)$ indicate the plane of transverse sections in $(B, F$, and insets in $C, D, G$ and H). (I-L) Expression of is/2b:Gfp at 22 hpf (I, J) and neurog 1 at $30 \mathrm{hpf}(\mathrm{K}, \mathrm{L})$ in a specimen in which mature (fgf5-expressing) neurons were laser-ablated. The same specimen is shown in all panels. Mature SAG neurons expressing is/2b:Gfp were serially ablated on the left side at $22 \mathrm{hpf}(\mathrm{J})$ and $25 \mathrm{hpf}$ (not shown), and the embryo was fixed and sectioned at $30 \mathrm{hpf}$ to examine neurog1 expression (L). Images of the unablated right side (l, K) were inverted to facilitate comparison. The surface of the otic vesicle is outlined in all panels. Arrows in sections indicate the edges of neurog1 domain in the otic floor. Note that the amount and duration of delamination of neurog $1^{+}$neuroblasts is strongly enhanced by knockdown of $f g f 5(F, G)$ or ablation of mature neurons (L). Activation of $h s: f g f 8$ reverses the effects of $f g f 5-\mathrm{MO}(\mathrm{H})$. Scale bar, $25 \mu \mathrm{m}$. Transverse sections are shown with lateral to the left and dorsal up. Wholemount images show dorsolateral views with anterior to the left. doi:10.1371/journal.pgen.1003068.g006
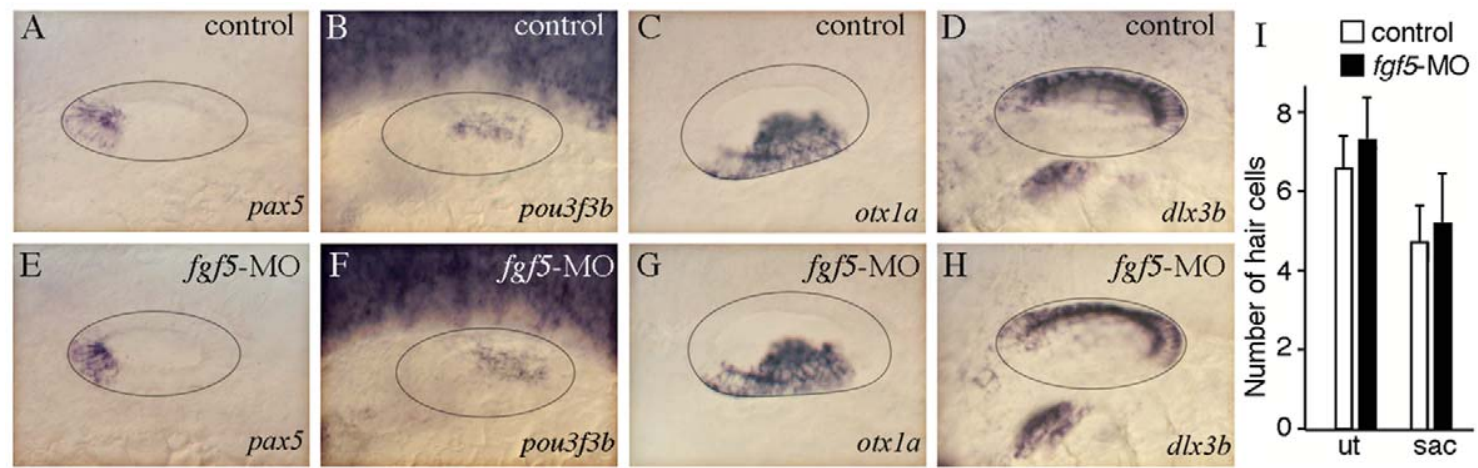

Figure 7. Normal axial patterning in fgf5 morphants. (A-H) Expression of regional patterning markers in control embryos (A-D) and fgf5 morphants $(\mathrm{E}-\mathrm{H})$. Expression of pax5 $(\mathrm{A}, \mathrm{E})$ and pou3f3b (B, F) labels anterior and posterior regions, respectively. Expression of otx $1 a(\mathrm{C}, \mathrm{G})$ and $d l \times 3 b$ $(\mathrm{D}, \mathrm{H})$ labels ventromedial and dorsolateral regions, respectively. The otic vesicle is outlined. Images show dorsolateral views with anterior to the left. (I) The total number of hair cells in the utricular (ut) and saccular (sac) maculae of control embryos and fgf5 morphants at $32 \mathrm{hpf}$. Data were obtained by counting GFP-positive hair cells (mean of total number \pm standard deviation) in the sensory epithelia of brn3c:Gfp transgenic embryos. Data show means and standard deviations from 20 specimens each. Differences between control and experimental specimens were not statistically significant $(p=0.16$ for the utricle, $p=0.67$ for the saccule) based on Student's $t$ tests.

doi:10.1371/journal.pgen.1003068.g007 


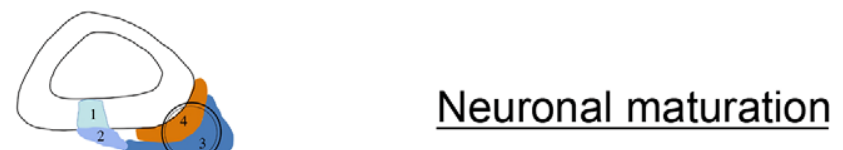

I. $\uparrow$ or $\downarrow$ Fgf at $24 \mathrm{hpf}$, examine neurod \& Isl1 at 36, $48 \mathrm{hpf}$.
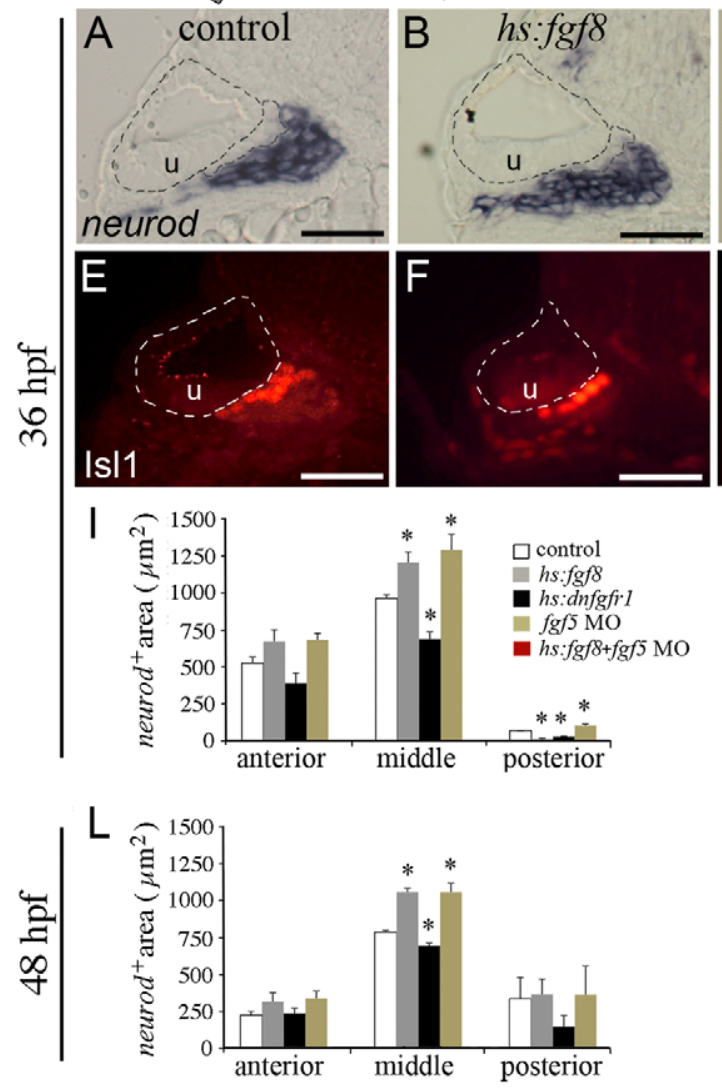
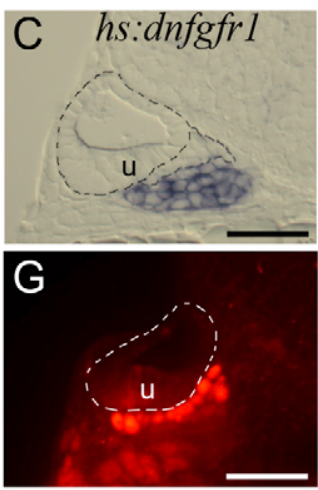

$\mathrm{J}$
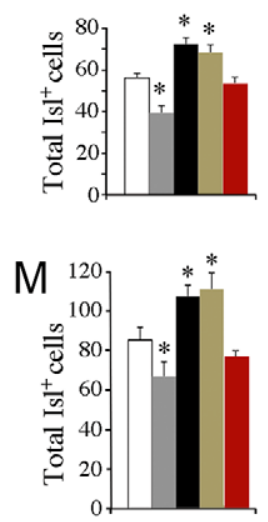

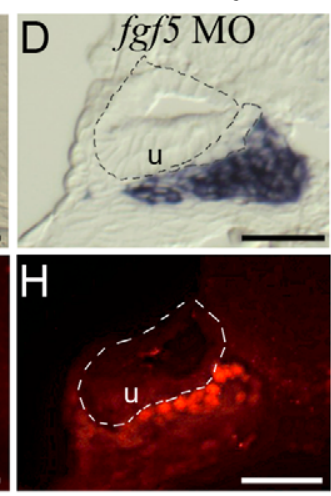

$\mathrm{K}$

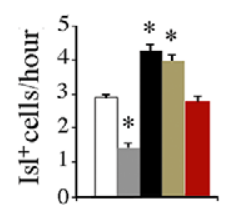

$\mathrm{N}$

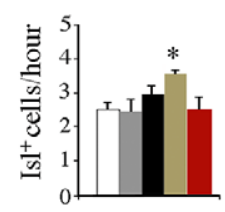

Figure 8. Fgf regulates the balance between transit-amplification and differentiation. The icon at the top of the figure indicates that neuronal maturation (neurod ${ }^{+}$transit-amplifying cells and $|s| 1^{+}$mature neurons) is the focus of analysis. Manipulations in these experiments (Neuronal maturation group I) are briefly summarized at the top. Embryos were heat shocked for 30 minutes at $39^{\circ} \mathrm{C}$ (wild-type controls, hs:fgf8/+ embryos, and fgf5-morphants) or $38^{\circ} \mathrm{C}$ (hs:dnfgfr1/+ embryos) beginning at $24 \mathrm{hpf}$. (A-H) Transverse sections (lateral to the left, dorsal up) showing neurod expression (A-D) or Isl1 staining (E-H) at 36 hpf in control embryo (A, E), hs:fgf8/+ embryos (B, F), hs:dnfgfr 1/+ embryos (C, G) and fgf5 morphants (D, H). All sections shown pass through the middle region of the SAG at the level of the utricular macula $(u)$. The otic vesicle is outlined. Scale bar, $25 \mu \mathrm{m}$. (I-N) Quantitation of transit-amplifying and mature neuronal populations at $36 \mathrm{hpf}(\mathrm{I}-\mathrm{K})$ and at $48 \mathrm{hpf}(\mathrm{L}-\mathrm{N})$. Panel I shows a color key to facilitate comparison between treatments: White bars, control; gray bars, hs:fgf8; black bars, hs:dnfgfr 1; brown bars, fgf5 morphants; red bars, activation of hs:fgf8 in fgf5 morphants. Analysis of transverse sections was used to measure the mean area of neurod ${ }^{+}$precursor cells $(\mathrm{I}, \mathrm{L})$ in the anterior, middle and posterior regions of SAG. The total number of Isl1+ neurons $(\mathrm{J}, \mathrm{M})$ and the mean hourly rate of neuron production from $24 \mathrm{hpf}$ to $36 \mathrm{hpf}(\mathrm{K})$ and from 36 to $48 \mathrm{hpf}(\mathrm{N})$ was measured by counting neurons in stained wholemount specimens. Error bars in I, J, L, M indicate standard deviations $(\mathrm{n}=3$ or greater for sectional areas; $\mathrm{n}=15$ for $|\mathrm{s}| 1^{+}$cell counts). ${ }^{*} \mathrm{p}<0.05$ in comparison to control, analyzed with Student's $t$ test. Error bars in $\mathrm{K}, \mathrm{N}$ indicate standard errors.

doi:10.1371/journal.pgen.1003068.g008

proportion of cells incorporating BrdU (data not shown), indicating that Fgf does not directly affect cell cycle dynamics. Instead, the data (summarized in Table 2) suggest that Fgf slows the rate at which transit amplifying cells differentiate into mature SAG neurons, whereas blocking Fgf accelerates differentiation.

We next assessed the role of Fgf5 in restraining maturation of precursor cells. In $f g f 5$ morphants, the size of neurod ${ }^{+}$domain was increased in both the middle and posterior regions of SAG in the embryos at 36 and $48 \mathrm{hpf}$ (Figure 8D, 8I, 8L). Note that the increase in the transit-amplifying region seen in $f g f 5$ morphants was different from what was observed following activation of $h s: d n f g f r 1$. This is presumably because the prolonged phase of robust specification seen in $f g f 5$ morphants (Figure 6G, 6H) continues to replenish the transit-amplifying population. Addi- tionally, $f g f 5$ morphants also produced more $\mathrm{Isll}^{+}$neurons than normal (Figure $8 \mathrm{H}, 8 \mathrm{~J}, 8 \mathrm{~K}, 8 \mathrm{M}, 8 \mathrm{~N}$ ). However, despite the enlarged pool of precursors $f g f 5$ morphants did not produce more mature neurons than did hs:dnfgfrl embryos (Figure 8J, 8M). This is possibly because redundant factors (possibly macular Fgfs) continue to restrain the enlarged pool of progenitors in $f g f 5$ morphants. Finally, we observed that strong activation of $h s: f g f o$ $\left(39^{\circ} \mathrm{C}\right)$ at $24 \mathrm{hpf}$ in $f g f 5$-morphants restored neuron production to normal (Figure 8J, 8K, 8M, 8N). Thus, as during neuroblast specification, the rate of neuronal maturation is also regulated by a proper balance of Fgf signaling. Moreover, these data (summarized in Table 2) support a role for Fgf5 as a feedback inhibitor released by mature SAG neurons to restrict the rate of neuronal differentiation. 
Table 2. Effects of altering Fgf on SAG maturation.

\begin{tabular}{|c|c|c|c|}
\hline Condition and stage & Size neurod domain & Number IsI1 ${ }^{+}$cells & Figure \\
\hline hs:fgf8-high $\left(39^{\circ} \mathrm{C}\right), 24 \mathrm{hpf}$ & increased $36,48 \mathrm{hpf}$ & reduced $36,48 \mathrm{hpf}$ & $8 \mathrm{~B}, 8 \mathrm{~F}, 81-8 \mathrm{~N}$ \\
\hline hs:dnfgfr1-high $\left(38^{\circ} \mathrm{C}\right), 24 \mathrm{hpf}$ & reduced $36,48 \mathrm{hpf}$ & increased 36, $48 \mathrm{hpf}$ & $8 \mathrm{C}, 8 \mathrm{G}, 8 \mathrm{l}-8 \mathrm{~N}$ \\
\hline fgf5-MO, 1-cell & increased $36,48 \mathrm{hpf}$ & increased 36, $48 \mathrm{hpf}$ & $8 \mathrm{D}, 8 \mathrm{H}, 8 \mathrm{I}-8 \mathrm{~N}$ \\
\hline fgf5-MO+hs:fgf8 $\left(39^{\circ} \mathrm{C}\right), 24 \mathrm{hpf}$ & not determined & normal 36, $48 \mathrm{hpf}$ & $8 J, 8 \mathrm{~K}, 8 \mathrm{M}, 8 \mathrm{~N}$ \\
\hline Ablate mature SAG $30,32 \mathrm{hpf}$ & reduced $44 \mathrm{hpf}$ normal $56 \mathrm{hpf}$ & normal 34-44 hpf* increased 56-80 hpf* & $9 \mathrm{~A}$ \\
\hline hs:fgf8-high $\left(39^{\circ} \mathrm{C}\right), 34 \mathrm{hpf}$ & normal $56 \mathrm{hpf}$ & reduced $34-56 \mathrm{hpf}^{*}$ & $9 B$ \\
\hline hs:dnfgfr 1-high $\left(38^{\circ} \mathrm{C}\right), 34 \mathrm{hpf}$ & normal $56 \mathrm{hpf}$ & increased 34-56 hpf* & $9 B$ \\
\hline Ablate SAG+hs:fgf8 $\left(39^{\circ} \mathrm{C}\right), 34 \mathrm{hpf}$ & normal $56 \mathrm{hpf}$ & normal $34-56 \mathrm{hpf}^{*}$ & $9 B$ \\
\hline Ablate SAG+hs:dnfgfr $1\left(38^{\circ} \mathrm{C}\right), 34 \mathrm{hpf}$ & normal 56 hpf & increased 34-44 hpf* & $9 B$ \\
\hline
\end{tabular}

*Rate of mature neuron production during the indicated interval.

doi:10.1371/journal.pgen.1003068.t002

\section{Neuronal maturation following ablation of mature SAG neurons}

To further explore regulation of SAG maturation, we assessed whether laser-ablation of mature SAG neurons affects the rate of new neuron production. This analysis was conducted after $30 \mathrm{hpf}$ to minimize the impact of neuroblast specification on overall cell number. Using the isl2b:Gfp line, mature SAG neurons on one side of the head were targeted for serial ablation at $30 \mathrm{hpf}$ and $32 \mathrm{hpf}$, with the contralateral side serving as a non-ablated control. We observed that a single round of ablation was inefficient, allowing a substantial fraction of neurons to survive. However, serial ablation successfully eliminated over $90 \%$ of mature neurons, as confirmed by anti-Isll staining just after the second ablation (not shown). Analysis of the transit-amplifying population revealed that the number of neurod ${ }^{+}$cells declined by $10-20 \%$ on the ablated side during the first 12 hours following neuronal ablation, probably reflecting collateral damage, but the number returned to normal by $56 \mathrm{hpf}$ ( 24 hours post-ablation) (data not shown). Despite the initial decrease in transit-amplifying cells, new $\mathrm{Isll}^{+}$neurons accumulated at a rate comparable to the non-ablated contralateral side for the first 12 hours after ablation, (Figure 9A). The rate of neuron production briefly declined during the next 12-hour period, but then increased to a rate $60 \%$ greater than normal through at least $80 \mathrm{hpf}$ (Figure 9A, Table 2). Co-ablation of both mature and transit-amplifying cells (the latter were targeted based on position and morphology) nearly eliminated production of new $\mathrm{Isll}^{+}$cells through at least $56 \mathrm{hpf}$ (Figure 9A), confirming the vital importance of transit-amplifying cells for producing new mature SAG neurons. Together these data suggest that loss of feedback inhibition from mature neurons leads to accelerated differentiation of cells from a pool of selfrenewing progenitors.

We next examined whether altering Fgf signaling influences neuron production after $30 \mathrm{hpf}$, with and without laser-ablation of mature neurons. Ablations were conducted in transgenic embryos carrying both isl2b:Gfp and either $h s: f g f o$ or $h s: \operatorname{dnfgfr} 1$. Again, is $2 \mathrm{~b}: G \mathrm{fp}^{+}$cells were serially ablated on one side at $30 \mathrm{hpf}$ and $32 \mathrm{hpf}$, and embryos were then heat shocked at $38^{\circ} \mathrm{C}$ or $39^{\circ} \mathrm{C}$ (strong activation) at $34 \mathrm{hpf}$. The contralateral side served as a non-ablated control. On the non-ablated side, the effects of activating $h s: f g f o$ or $h s: \operatorname{dnfg} g r 1$ at $34 \mathrm{hpf}$ were similar the effects of activating these transgenes at $24 \mathrm{hpf}$ : Specifically, strongly elevating Fgf impaired production of new neurons whereas blocking Fgf accelerated production of new neurons (Figure 9B, Table 2). On the ablated side, activation of $h s: \operatorname{dnfg} g r 1\left(38^{\circ} \mathrm{C}\right)$ accelerated production of new neurons to more than twice the normal rate through $44 \mathrm{hpf}$, after which the rate flattened out as in non-transgenic ablations (Figure 9B). Moreover, the rate of neuron production in ablated $h s: d n f g f r 1$ embryos was $50 \%$ greater than in non-ablated $h s: \operatorname{dnfg} f r 1$ embryos. Surprisingly, strong activation of $h s: f g f o\left(39^{\circ} \mathrm{C}\right)$ resulted in a rate of neuronal accumulation on the ablated side that was nearly normal (comparable to the nonablated control). Thus, misexpressing Fgfo counterbalances the effects of eliminating mature neurons (and hence Fgf5) such that there is no net change in the rate of neuron production. This is similar to the ability of $h s: f g f o$ to counterbalance the effects of $f g f 5$ $\mathrm{MO}$ on neuroblast specification (Figure $6 \mathrm{H}$ ) and maturation of SAG neurons (Figure 8J, 8K, 8M, 8N). Analysis of the neurod ${ }^{+}$ domains showed that transgene activity had no significant effect on the size of the transit-amplifying pool at these stages (Table 2). Thus, blocking Fgf accelerates production of new neurons and enhances the effects of neuronal ablation whereas misexpressing

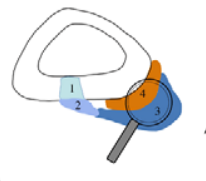

\section{Neuronal maturation}

II. Ablate mature neurons at $30,32 \mathrm{hpf}$; 4or $\downarrow$ Fgf at $34 \mathrm{hpf}$, examine Isl1 at 44, $56 \mathrm{hpf}$.
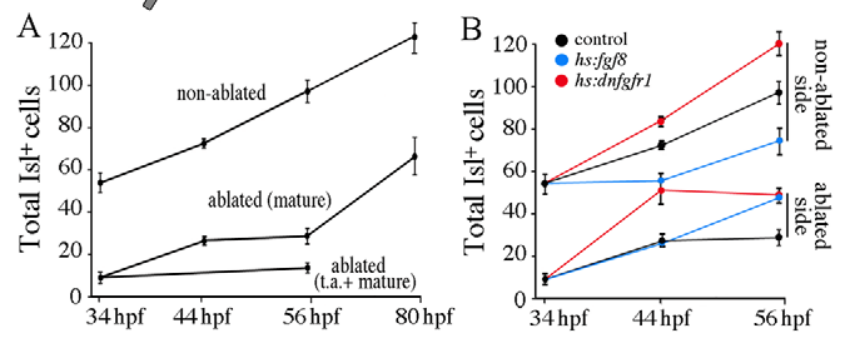

Figure 9. Regeneration following SAG ablation. The icon at the top of the figure indicates that neuronal maturation is the focus of analysis. Manipulations in these experiments (Neuronal maturation group II) are briefly summarized at the top. (A) Accumulation of $I \mathrm{~s}^{+}{ }^{+}$ SAG neurons in is/2b:Gfp/+ embryos after serial ablation of Gfp-positive neurons (mature) or ablation of Gfp-positive neurons and transitamplifying cells (t.a. + mature) at $30 \mathrm{hpf}$ and $32 \mathrm{hpf}$. Neuronal accumulation on the contralateral (non-ablated) side served as a control. (B) Effects of modulating Fgf after serial ablations at $30 \mathrm{hpf}$ and $32 \mathrm{hpf}$ on the total number of $|\mathrm{s}| 1^{+}$neurons. Embryos were heat shocked for 30 minutes at $39^{\circ} \mathrm{C}\left(+/+\right.$ and $h s: f g f 8 /+$ embryos) or $38^{\circ} \mathrm{C}$ (hs:dnfgfr1/+ embryos) beginning at $34 \mathrm{hpf}$. Data show means and standard deviations of 2-5 specimens per time point. doi:10.1371/journal.pgen.1003068.g009 
Fgf8 offsets the effects of neuronal ablation (summarized in Table 2). These data further support the hypothesis that Fgf5 from mature neurons acts as a feedback inhibitor to slow the rate of maturation of new SAG neurons.

\section{Discussion}

The data presented here support a model in which changing levels of Fgf differentially regulates distinct stages of SAG development (Figure 10). Initially a moderate level of Fgf in a spatial gradient specifies the neurogenic domain within the otic vesicle. Subsequently, Fgf levels gradually rise as differentiating SAG neurons accumulate and express Fgf5, eventually terminating neurogenesis in the otic vesicle. It is likely that the expanding macular source of Fgf also contributes to termination of neurogenesis. Terminal differentiation of SAG neurons initially occurs rapidly following delamination from the otic vesicle. However, the developmental increase in Fgf delays neuronal differentiation, maintaining the transit-amplifying phase. This allows the developing SAG to achieve a steady state in which the rate of progenitor growth just matches the rate of neuronal differentiation. This property of the SAG is presumably necessary to provide sufficient neurons to innervate growing sensory epithelia, which continue to expand throughout larval and early adult stages in zebrafish [30]. Knockdown of $f g f 5$ prolongs the phase of neuroblast specification and also accelerates the rate of neuronal differentiation. Neuroblast specification eventually ceases in $f g f 5$

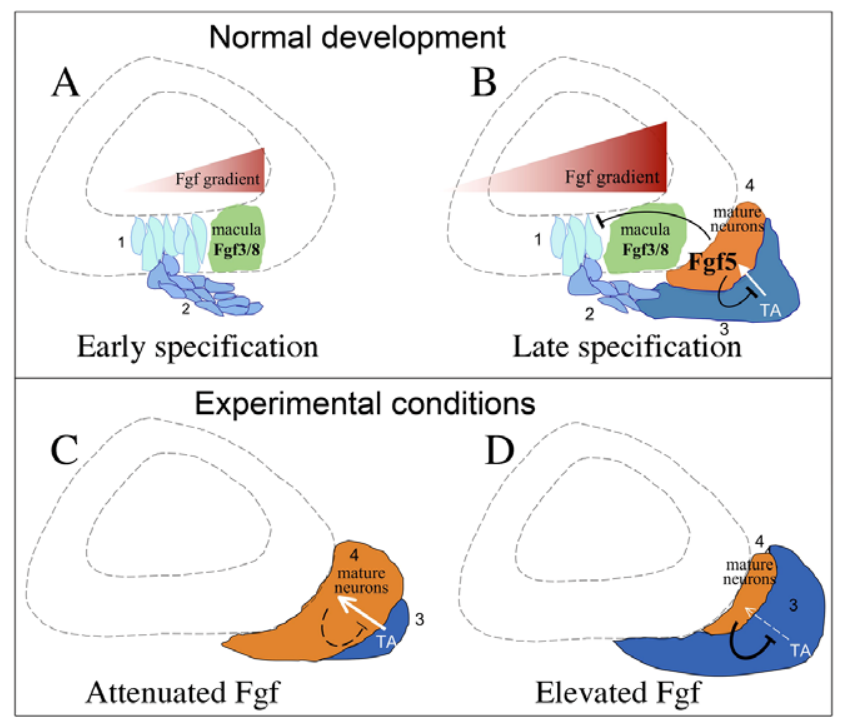

Figure 10. Model for regulation of SAG development by Fgf. (A) Neuroblast specification at early stages. A moderate level of Fgf3 and Fgf8 in a gradient generated by the utricular macula specifies neuroblasts in the floor of the otic vesicle (step 1), and nascent neuroblasts quickly delaminate from the otic vesicle (step 2). (B) As development proceeds, neuroblasts establish a pool of transit-amplifying (TA) progenitors (step 3), which eventually differentiate into mature neurons and express Fgf5 (step 4). Rising levels of neuronal Fgf5, combined with Fgf3 and Fgf8 from the growing utricular macula, exceeds an upper threshold that serves to terminate specification of new neuroblasts within the otic vesicle. Neuronal Fgf5 also slows differentiation of progenitors into mature neurons. $(C, D)$ At stages immediately following establishment of the transit-amplifying pool, experimental attenuation of Fgf signaling promotes maturation of neurons at the expense of progenitors (C) whereas elevating Fgf inhibits maturation, expanding the size of the transit-amplifying pool (D).

doi:10.1371/journal.pgen.1003068.g010 morphants, possibly in response to elevated Fgf from the growing utricular macula. We cannot assess the long-term effects of $f g f 5$ knockdown because morpholino efficacy dissipates after 3-5 days. However, once specification/delamination ceases, accelerated neural differentiation in the absence of $f f f 5$ function would be expected to deplete the transit-amplifying pool, leading to a neural deficiency in the long-run.

Cell cycle dynamics are likely to be rather complex in the transit-amplifying population. With a steady state of 180-200 progenitors producing 50-60 neurons per day, the average cell cycle length could be unusually long assuming that most progenitors continue to cycle. However, patterns of BrdU incorporation suggest cell cycle dynamics are not uniform amongst progenitors. A 6-hour incorporation period labels roughly $40 \%$ of neurod ${ }^{+}$progenitors, with labeling being especially prominent in distal regions of the SAG (Figure $1 \mathrm{E}, 1 \mathrm{~F}$, and data not shown). It is therefore possible that a substantial fraction of progenitors enter a quiescent state. We detected no overt effect of Fgf signaling on the pattern of BrdU incorporation or the incidence of mitotic cells (not shown), although we cannot exclude the possibility that our heat shock lines act too transiently to detectably alter cell cycle dynamics. Nevertheless, modulating Fgf clearly had a rapid effect on the rate of production of mature neurons (Figure 8 and Figure 9), thereby indirectly affecting the progenitor pool. Interestingly, activating $h s: f g f 8$ or $h s: d n f g f r 1$ at $24 \mathrm{hpf}$ caused lasting changes in the size of the progenitor pool (Figure 8), whereas no such change was seen when the transgenes were activated at $34 \mathrm{hpf}$ (data not shown). This difference likely reflects the greater relative impact of changing the rate of neuronal differentiation at early stages when the progenitor pool is still small. In contrast, with nearly 200 progenitors at $32 \mathrm{hpf}$, it is not surprising that altering the rate of neuronal differentiation by $1-2$ cells per hour for several hours had little impact on the progenitor pool.

Although the model in Figure 10 depicts the influence of macular Fgfs on SAG specification in the otic vesicle, it must be emphasized that a lateral gradient of Fgf from the hindbrain acts much earlier to coordinate formation of sensory and neural progenitors in adjacent domains during placodal stages. The prosensory gene atoh $1 b$ is induced at $10.5 \mathrm{hpf}$ in the nascent otic placode in medial cells closest to the Fgf-source [13], and elevating Fgf expands this domain laterally (our unpublished observations). Expression of neurog1 begins in more lateral tissue by $16 \mathrm{hpf}$ (14 somites) and is initially influenced by the same hindbrain source of Fgf $[15,16]$. We have confirmed here that neuroblast specification requires Fgf whereas excess Fgf inhibits neurog1 expression, consistent with the notion that the neurogenic domain is established by intermediate levels of Fgf in a diffusion gradient. After formation of the otic vesicle, the utricular macula provides the strongest source of Fgf in the otic vesicle and the neurogenic domain forms an arc wrapping around the lateral and posterior edges of this source [20]. Later the saccular macula begins to expand and expresses more Fgf [13,31], with a corresponding posterior extension of the neurogenic domain to form a narrow band just lateral to the saccule [20]. Soon thereafter Fgf levels exceed the upper threshold and terminate neuroblast specification.

In zebrafish, it is currently unclear whether the spatial distribution of neuroblasts within the otic vesicle is directly tied to later distribution of mature neurons in the SAG. However, fate mapping studies in chick and mouse reveal that the spatialtemporal progression of neuroblast formation in the otic vesicle presages the spatial-temporal accumulation of vestibular and auditory neurons outside the ear [32,33]. It is likely that a similar 
progression occurs in zebrafish, though fate mapping studies have yet to confirm this relationship. Mechanism of SAG subtype specification has not been well characterized in any species, though several studies in zebrafish suggests that Shh is involved. Disruption of Hedgehog signaling ablates posterior fates in the otic vesicle, including the saccule and posterior (presumptive auditory) neurons [23,34]. Fgf signaling acts in opposition to Shh by promoting anterior fates in the otic vesicle [15,16,34], though altering Fgf does not appear to cause wholesale redistribution of SAG neurons. However, we found that activating $h s: f g f o$ at $24 \mathrm{hpf}$ caused premature termination of neuroblast specification and also blocked later production of posterior/auditory neurons, supporting a link between spatial/temporal cues and SAG subtypespecification.

\section{Are the distinct roles of Fgf conserved in amniotes?}

Numerous studies support a role for Fgf in SAG neuroblast specification in the chick and mouse. In chick, misexpression of Fgf8 or Fgf10 during placodal stages causes expansion of the neurogenic domain in the otic vesicle, whereas blocking Fgf signaling dramatically reduces the neurogenic domain $[4,35]$. In mouse, knockout of Fgf3 or receptor isoform Fgfr-2 (IIIb) causes severe deficiencies of delaminating neuroblasts and neurons $[11,36]$. Explant cultures of chick or mouse otocysts treated with exogenous Fgf2 produce 5- to 10-fold more delaminated neuroblasts compared to controls, whereas blocking Fgf2 with a neutralizing antibody severely reduces the number of neuroblasts $[10,37]$. Thus the requirement for Fgf in neuroblast specification appears broadly conserved. However, the spatial gradient of Fgf that we propose coordinates sensory and neural development in zebrafish is unlikely to operate in mammals. Unlike the situation in zebrafish, in mouse the neurogenic and sensory domains overlap spatially but are specified at slightly different times. Neuroblast specification occurs first, but as the phase of neuroblast specification/delamination begins to wane sensory epithelia begin to form in the same region. The transition from neural to sensory development partly reflects mutual repression between Neurogl and Atohl, the principal initiators the proneural and prosensory pathways, respectively [38]. Whether Fgf also influences this transition is not known.

Despite the above studies showing a requirement for Fgf, it is not clear whether high levels of Fgf are inhibitory in birds and mammals as we have shown here, nor whether Fgf delays maturation of cells in the transit-amplifying pool. In explants of chick or mouse otocysts, exposure to Fgf accelerates the appearance of mature neurons compared to cultures lacking exogenous Fgf [10,37]. At first glance, these results appear to contradict our findings that Fgf delays differentiation. However, Fgf levels used in the above explant studies were based on doseresponse curves and were selected to optimize growth of the explant. Hence potential inhibitory effects of higher doses of Fgf were not evaluated. Furthermore, neuroblasts in culture disperse after delamination rather than accumulating against the otocyst wall where they might facilitate feedback inhibition. This possibly explains why otic explants continue to produce neuroblasts for many days, far longer than during normal embryonic development. In rodent embryos, differentiating auditory neurons express Fgf1, Fgf2, Fgf5 and Fgf10 [36,39-42], which could help mediate feedback inhibition. Unfortunately, relevant functional studies are lacking. In adult rodents, neuronal Fgf is thought to play a role in maintenance of the spiral ganglion. Augmenting Fgf mitigates neural degeneration following nerve injury or noiseinduced trauma $[43,44]$. Additionally, conditional knockout of Fgf receptor genes Fgfr 1 and Fgfr2 in glial cells in the spiral ganglion leads to progressive loss of auditory neurons beginning around 2 months of age, suggesting a role in promoting trophic support from glia [45]. In cultures of spiral ganglion from adult mouse, exogenous Fgf2 can promote neuronal survival and neurite outgrowth [46]. Unexpectedly, such cultures were also found to contain quiescent progenitors that could be induced to reenter the cell cycle by incubation with EGF and Fgf2, with some cells differentiating into neurons after removal of EGF and Fgf2 [46]. These latter data are consistent with the possibility that Fgf maintains progenitors and inhibits neural differentiation, though it remains to be seen whether such a mechanism operates in vivo.

The developing SAG can be compared to the developing olfactory epithelium (OE). Fgf8 expression around the rim of the olfactory pit stimulates proliferation of $\mathrm{OE}$ progenitors, which differentiate into mature neurons deeper inside the pit away from the Fgf8 source [47]. Conditional knockout of Fgf8 results in severe deficiency of neurons due to failure of progenitors to expand. Development of the OE neurons is also regulated by feedback inhibition from mature neurons, though the mechanism differs from the SAG. Specifically, mature OE neurons secrete the TGF $\beta$ factor GDF11, which inhibits further proliferation of progenitors by antagonizing Fgfo [48]. In the eye, too, GDF11 acts as a feedback inhibitor of retinal ganglion cells, though in this case GDF11 blocks further differentiation of progenitors rather than restricting proliferation [49].

In numerous other settings, Fgf regulates the balance between growth and differentiation of neural progenitors. In cultures of human or rat cortical progenitors, high levels of Fgf stimulate proliferation and block neuronal differentiation [50,51]. In the developing midbrain-hindbrain region in mouse, conditional knockdown of Fgf receptors results in an increase in differentiated neurons and a concomitant loss of progenitor cells in the ventricular zone [52]. During earlier stages of mouse development, Fgf induces embryonic stem (ES) cells to form epiblast, which begin to express Fgf5. Subsequently, Fgf maintains the epiblast as a stable intermediate by preventing reversion back to the ES ground state and blocking further differentiation into neural ectoderm [53]. Thus, maintenance of stable progenitor pools by Fgf appears to be a broadly conserved mechanism utilized in many aspects of neural development. A relatively novel aspect of SAG development is that Fgf coordinates the entire process, initially specifying neuroblasts and, at a higher level, also mediates feedback from mature neurons to inhibit further differentiation. How changing levels of Fgf achieve this balance remains an important unresolved question.

\section{Materials and Methods}

\section{Fish strains, misexpression, and inhibitor treatment}

Wild-type zebrafish strains were derived from the $\mathrm{AB}$ line (Eugene OR). The following transgenic lines were used in this study: $\operatorname{Tg}(h s p 70: f g f \delta)^{x 17}$ [24], $\operatorname{Tg}(h s p 70 I: \operatorname{dnfg} f r 1-E G F P)^{p d 1}$ [25] and $\mathrm{Tg}(-17.6 i s l 2 b: G F P)^{z c 7}$ [22]. Embryos were maintained at $28^{\circ} \mathrm{C}$, unless otherwise stated, and staged according to standard protocol [54]. Heat shock-inducible transgenes were activated by incubating embryos for 30 minutes at elevated temperatures as indicated in the Results. In some experiments, Fgf signaling was blocked by treating wild-type embryos in their chorions with SU5402 (Tocris Bioscience) diluted from a $20 \mathrm{mM}$ stock in DMSO to a final concentration of $100 \mu \mathrm{M}$ SU5402. PTU (1-phenyl 2-thiourea, $0.3 \mathrm{mg} / \mathrm{ml}$, Sigma) was added to fish water to prevent melanin formation. 


\section{Morpholino injection and RT-PCR}

To block $f f f 5$ translation, we used $f f f t b$-MO" 5 -CATTCTTTCCAGAGAGCGCTAGGCG-3'. To block splicing of $f \circ f 5$ transcript, we used fof5ile2-MO: 5' -GCTCCAGCACACCTAGATAGAGAAA- 3'. Approximately $5 \mathrm{ng}$ morpholino was injected per embryo at one-cell stage. Both morpholinos gave identical phenotypes. The efficacy of the splice blocker was assessed at $24 \mathrm{hpf}$ by RT-PCR with primers P1 (forward), 5'-TCGATGGAAGAGTCAACGGGAGG-3' and P2 (reverse) 5'-GCCTTCGCCTCTTGTTCATGGC-3' (see Figure 4D, 4E). Expression of ormithine decarboxylase (odc) was measured as a constitutive control. Uninjected embryos from the same genetic background were used to measure control transcript levels.

\section{In situ hybridization}

Whole-mount in situ hybridization was carried out with methods described previously $[55,56]$. A shorter riboprobe was synthesized for neurog1 using T7 RNA polymerase to avoid binding to shared vector sequences encoded by the $T g(-17.6 i s l 2 b: G F P)^{z c 7}$ transgene. To improve signal and reduce background staining during in situ hybridization for $f g f 5$, pre-hybridization and hybridization were performed at $70^{\circ} \mathrm{C}$ for 12 hours and 24 hours, respectively.

\section{Immunostaining}

Antibody staining was performed as described previously [57]. Primary antibodies were as follows: anti-Islet1/2 (Developmental Studies Hybridoma Bank 39.4D5, 1:100 for whole-mount, 1:250 for cryosections) and anti-BrdU (Beckton-Dickinson, 1:300). Secondary antibodies were as follows: HRP-conjugated goat anti-mouse IgG (Vector Labs PI-2000, 1:200) and Alexa 546 goat anti-mouse IgG (Invitrogen A-11003, 1:250).

\section{Cryosectioning and BrdU labeling}

Fixed embryos were washed three times for $5 \mathrm{~min}$ each in $1 \times$ PBS and then soaked in $20 \%$ sucrose solution made in PBS

\section{References}

1. Andermann P, Ungos J, Raible DW (2002) Neurogenin1 defines zebrafish cranial sensory ganglia precursors. Dev Biol 251: 45-58.

2. Ma Q Chen Z, del Barco Barrantes I, de la Pompa JL, Anderson DJ (1998) neurogenin1 is essential for the determination of neuronal precursors for proximal cranial sensory ganglia. Neuron 20: 469-482.

3. Korzh B, Sleptsova I, He J, Gong Z (1998) Expression of zebrafish bHLH genes ngn 1 and nrd defines distinct stages of neural differentiation. Dev Dyn 213: 92-104.

4. Alsina B, Abelló G, Ulloa E, Henrique D, Pujades C, et al. (2004) FGF signaling is required for determination of otic neuroblasts in the chick embryo. Dev Biol 267: 119-134.

5. Begbie J, Ballivet M, Graham A (2002) Early steps in the production of sensory neurons by the neurogenic placodes. Mol Cell Neurosci 21: 502-511.

6. D'Amico-Martel A (1982) Temporal patterns of neurogenesis in avian cranial sensory and autonomic ganglia. Am J Anat 163: 351-372.

7. Matei V, Pauley S, Kaing S, Rowitch D, Beisel KW, et al. (2005) Smaller inner ear sensory epithelia in Neurog1 null mice are related to earlier hair cell cycle exit. Dev Dyn 234:3 89-395.

8. Camarero G, Leon Y, Gorospe I, De Pablo F, Alsina B, et al. (2003) Insulin-like growth factor 1 is required for survival of transit-amplifying neuroblasts and differentiation of otic neurons. Dev Biol 262: 242-253.

9. Adamska M, Herbrand H, Adamski M, Kruger M, Braun T, et al. (2001) FGFs control the patterning of the inner ear but are not able to induce the full ear program. Mech Dev 109: 303-319.

10. Hossain WA, Zhou X, Butledge A, Baier C, Morest DK (1996) Basic Fibroblast Growth Factor affects neuronal migration and differentiation in normotypic cell cultures from the cochleovestibular ganglion of the chick embryo. Exp Neurol 138: 121-143.

11. Mansour SL, Goddard JM, Capecchi MR (1993) Mice homozygous for a targeted disruption of the proto-oncogene int-2 have developmental defects in the tail and inner ear. Development 117: 13-28.

12. Léger S, Brand M (2002) Fgf8 and Fgf3 are required for zebrafish ear placode induction, maintenance and inner ear patterning. Mech Dev 119: 91-108.

13. Millimaki BB, Sweet EM, Dhason MS, Riley BB (2007) Zebrafish atohl genes: classic proneural activity in the inner ear and regulation by Fgf and Notch. Development 134: 295-305. followed by $30 \%$ sucrose until sinking to the bottom of a microcentrifuge tube. Embryos were embedded in tissue freezing medium (Triangle Biomedical Sciences, TFM-G) and transverse sections were cut at $10 \mu \mathrm{m}$ thickness using a cryostat and immunostained. Finally, slides were washed twice in $1 \times$ PBS and mounted in ProLong Gold (Invitrogen) with a coverslip. For double labeling, whole-mount in situ hybridization was performed first followed by immunostaining on cryosections. For BrdU labeling, dechorionated embryos were incubated in fish water containing $10 \mathrm{mM} \mathrm{BrdU}$ in $1 \%$ DMSO for the indicated duration. Embryos were rinsed twice for 5 minutes each in fish water prior to fixation. For older stages (96 hpf) $2 \mathrm{nl}$ of $10 \mathrm{mM}$ BrdU/1\% DMSO solution with 3\% filtered green food coloring was injected into the brain ventricle of larvae anesthetized in Tricaine (Sigma). Embryos were first processed by whole-mount in situ hybridization for neurod and then cryosectioned. Slides were washed thrice for 5 minutes each in PBT (with $0.1 \%$ Triton) and incubated in $2 \mathrm{~N} \mathrm{HCl}$ for 45 minutes at $37^{\circ} \mathrm{C}$. Slides were rinsed in PBT again, incubated in blocking solution (with $1 \%$ Triton for $36 \mathrm{hpf}$ and $3 \%$ Triton for $102 \mathrm{hpf}$ ) for 2 hours and stained for BrdU.

\section{Laser ablation}

Maturing SAG neurons were ablated using a MicroPoint laser, under $40 \times$ objective, in isl2b:GFP transgenic line that labels this population of cells. Anesthetized embryos were mounted in a dorsolateral orientation beneath a \#1 coverslip on a bridge slide made by stacking two \#1 coverslips on either side of the embryo.

\section{Author Contributions}

Conceived and designed the experiments: SV HK BBR. Performed the experiments: SV HK MSP. Analyzed the data: SV HK MSP BBR. Contributed reagents/materials/analysis tools: BBR. Wrote the paper: SV BBR.

14. Adamska M, Léger S, Brand M, Bober E (2000) Expression of a new zebrafish inner ear and lateral line specific gene, Nkx5.1, is significantly reduced in the ears of Fgf8 mutant, ace. Mech Dev 97: 161-165.

15. Kwak SJ, Phillips BT, Heck R, Riley BB (2002) An expanded domain of $f g f 3$ expression in the hindbrain of zebrafish valentino mutants results in mispatterning of the otic vesicle. Development 129: 5279-5287.

16. Lecaudey V, Ulloa E, Anelme I, Stedman A, Schneider-Maunoury S, et al. 2007) Role of the hindbrain in patterning the otic vesicle: A study of the zebrafish vhnf1 mutant. Dev Biol 303: 134-143.

17. Alsina B, Giraldez F, Valera-Nieto I (2003) Growth factors and early development of otic neurons: Interactions between intrinsic and extrinsic signals. Curr Top Dev Biol 57: 177-206.

18. Sánchez-Calderón H, Milo M, León Y, Varela-Nieto I (2007) A network of growth and transcription factors controls neuronal differentiation and survival in the developing ear. Int J Dev Biol 51: 557-570.

19. Radosevic M, Robert-Moreno A, Coolen M, Bally-Cuif L, Alsina B (2011) Her9 represses neurogenic fate downstream of Tbx1 and retinoic acid signaling in the inner ear. Development 138: 397-408.

20. Haddon C, Lewis J (1996) Early ear development in the embryo of the zebrafish, Danio rerio. J Comp Neurol 365: 112-128.

21. Liu M, Pereira FA, Price SD, Chu MJ, Shope C, et al. (2000) Essential role of Beta2/NeuroD1 in development of the vestibular and auditory systems. Gene Dev 14: 2839-2854.

22. Pittman AJ, Law MY, Chien CB (2008) Pathfinding in a large vertebrate axon tract: isotypic interactions guide retinotectal axons at multiple choice points. Development 135: 2865-2871.

23. Sapède D, Pujades C (2010) Hedgehog signaling governs the development of otic sensory epithelium and its associated innervation in zebrafish. J Neurosci 30(10): 3612-3623.

24. Millimaki BB, Sweet EM, Riley BB (2010) Sox2 is required for maintenance and regeneration, but not initial development, of hair cells in the zebrafish inner ear. Dev Biol 338: 262-269.

25. Lee Y, Grill S, Sanchez A, Murphy-Ryan M, Poss KD (2005) Fgf signaling instructs position-dependent growth rate during zebrafish fin regeneration. Development 132: 5173-5183. 
26. Raible R, Brand M (2001) Tight transcriptional control of ETS domain factors Ern and Pea3 by Fgf signaling during early zebrafish development. Mech Dev 107: 105-117.

27. Roehl H, Nüsslein-Volhard (2001) Zebrafish pea3 and erm are general targets of FGF8 signaling. Curr Biol 11: 503-507.

28. Wright TJ, Mansour SL (2003) Fgf signaling in ear development and innervation. Curr Top Dev Biol 57: 225-259.

29. Padanad MS, Bhat N, Guo B, Riley BB (2012) Conditions that influence the response to Fgf during otic placode induction. Dev Biol 364: 1-10.

30. Higgs DM, Souza MJ, Wilkins HR, Presson JC, Popper AN (2001) Age- and size-related changes in the inner ear and hearing ability of the adult zebrafish (Danio rerio). JARA 03: 174-184.

31. Feng Y, Xu Q (2010) Pivotal role of $h m \times 2$ and $h m \times 3$ in zebrafish inner ear and lateral line development. Dev Biol 339: 507-518.

32. Koundakjian EJ, Appler JL, Goodrich LV (2007) Auditory neurons make stereotyped wireing decisions before maturation of their targets. J Neurosci 25: 14078-14088.

33. Bell D, Streit A, Gorospe I, Varela-Nieto I, Alsina B, Giraldez F (2008) Spatial and temporal segregation of auditory and vestibular neurons in the otic placode. Dev Biol 322: 109-120.

34. Hammond KL, Whitfield TT (2011) Fgf and Hh signaling act on a symmetrical pre-pattern to specify anterior and posterior identity in the zebrafish otic placode and vesicle. Development 138: 3977-3987.

35. Abelló G, Khatri S, Radosevic M, Scotting PJ, Giráldez F, et al. (2010) Independent regulation of Sox3 and $L m x 1 b$ by FGF and BMP signaling influences the neurogenic and non-neurogenic domains in the chick otic placode. Dev Biol 339: 166-178.

36. Pirvola U, Spencer-Dene B, Xing-Qun L, Kettunen P, Thesleff I, et al. (2000) FGF/FGFR-2(IIIb) signaling is essential for inner ear morphogenesis. J Neurosci 20(16): 6125-6134.

37. Hossain WA, Morest DK (2000) Fibroblast Growth Factor (FGF-1, FGF-2) promote migration and neurite growth of mouse cochlear ganglion cells in vitro: immunohistochemistry and antibody perturbation. J Neurosci Res 62: 40-55.

38. Raft S, Koundakjian EJ, Quinones H, Jayasena CS, Goodrich LV, et al. (2007) Cross-regulation of $\mathrm{Ngn} 1$ and Math1 coordinates the production of neurons and sensory hair cells during inner ear development. Development 134: 4405-4415.

39. Goldfarb M, Bates B, Drucker B, Hardin J, Haub O (1991) Expression and possible functions of FGF-5 gene. Ann NY Acad Sci 638: 38-52.

40. Luo L, Koutnouyan H, Baird A, Ryan AF (1993) Acidic and Basic FGF mRNA expression in the adult and developing rat cochlea. Hearing Res 69: 182-193.

41. Pauley S, Wright TJ, Pirvola U, Ornitz D, Beisel K, et al. (2003) Expression and function of FGF10 in mammalian inner ear development. Dev Dyn 227: 203-215.

42. Silva VA, Gomide VG, Chadi G (2005) Fibroblast Growth Factor-2 immunoreactivity is present in the central and peripheral auditory pathways of adult rats. J Morphol 265: 141-151.
43. Sekiya TS, Shimamura N, Yagihahsi A, Suzuki S (2003) Effect of topically applied basic Fibroblast Growth Factor on injured cochlear nerve. Neurosurgery 52: 900-907.

44. D'Sa C, Gross J, Francone VP, Morest DK (2007) Plasticity of synaptic endings in the cochlear nucleus following noise-induced hearing loss is facilitated in the adult FGF2 overexpressor mouse. Eur J Neurosci 26: 666-680.

45. Wang SJ, Furusho M, D'Sa C, Kueada S., Conti L, et al. (2009) Inactivation of Fibroblast Growth Factor Receptor signaling in myelinating glial cells results in significant loss of adult spiral ganglion neurons accompanied by age-related hearing impairment. J Neurosci Res 87: 3428-3437.

46. Wei D, Jin Z, Järlebark L, Scarfone E (2006) Survival, synaptogenesis, and regeneration of adult mouse spiral ganglion neurons in vitro. J Neurobiol 67: $108-122$.

47. Kawauchi S, Shou J, Santos R, Hébert JM, McCounnel SK (2005) Fg 8 expression defines a morphogenetic center required for olfactory neurogenesis and nasal cavity development in mouse. Development 132: 5211-5223.

48. Wu HH, Ivkovic S, Murray RC, Jaramillo S, Lyons KM, et al. (2003) Autoregulation of neurogenesis by GDF11. Neuron 37: 197-207.

49. Kim J, Wu HH, Lander AD, Lyons KM, Matzuk MM, et al. (2005) GDF11 controls the timing of progenitor cell competence in developing retina. Science 308: 1927-1930.

50. Tsai RYL, Kim S (2005) Fibroblast Growth Factor 2 negatively regulates the induction of neuronal progenitors from neural stem cells. J Neurosci Res 82: $149-159$.

51. Nelson AD, Svendsen CN (2006) Low concentrations of extracellular FGF-2 are sufficient but not essential for neurogenesis from human neural progenitors. Mol Cell Neurosci 33: 29-35.

52. Saarimäki-Vire J, Peltopuro P, Lahti L, Naserke T, Blak AA, et al. (2007) Fibroblast Growth Factor receptors cooperate to regulate neural progenitor properties in the developing midbrain and hindbrain. J Neurosci 27(32): 85818592.

53. Greber B, Wu G, Bernemann C, Joo JY, Han DW, et al. (2010) Conserved and divergent roles for FGF signaling in mouse epiblast stem cells. Cell Stem Cell 6: 215-226.

54. Kimmel CB, Ballard WW, Kimmel SR, Ullman B, Schilling TF (1995) Stages of embryonic development of the zebrafish. Dev Dyn 203: 253-310.

55. Jowett T, Yan YL (1996) Double fluorescent in situ hybridization to zebrafish embryos. Trends Genet 12: 387-389.

56. Phillips BT, Bolding K, Riley BB (2001) Zebrafish $f g f 3$ and $f g f o$ encode redundant functions required for otic placode induction. Dev Biol 235: 351-365.

57. Riley BB, Chiang MY, Farmer L, Heck R (1999) The delta gene of zebrafish mediates lateral inhibition of hair cells in the inner ear and is regulated by pax2.1. Development 126: 5669-5678. 